Pacific Journal of Mathematics

ON CERTAIN IWAHORI INVARIANTS IN THE UNRAMIFIED 


\title{
ON CERTAIN IWAHORI INVARIANTS IN THE UNRAMIFIED PRINCIPAL SERIES
}

\author{
MARK ReEder
}

\begin{abstract}
An affine Hecke algebra is additively the tensor product of a finite dimensional Hecke algebra with the coordinate ring $\Theta$ of a complex torus. In this paper we give explicit formulas for eigenvectors of $\boldsymbol{\theta}$ in unramified principal series representations of the reductive $p$-adic group $G$ associated to $\mathscr{H}$. This leads to new information about intertwining operators, Jacquet modules and submodules of principal series representations.
\end{abstract}

Let $G$ be a reductive $p$-adic group, $\tau$ an unramified character of a minimal parabolic subgroup $P$, and $I(\tau)=\operatorname{ind}_{P}^{G} \tau$ the induced principal series representation of $G$. The space $I(\tau)^{B}$ of vectors in $I(\tau)$ which are invariant under an Iwahori subgroup $B$ affords a representation of the affine Hecke algebra $\mathscr{H}$ corresponding to $G$. It is known that taking $B$-invariants yields an equivalence of categories between (admissible $G$-modules generated by their $B$-invariants) and (finite dimensional $\mathscr{H}$ modules). Thus the representation theory of $I(\tau)$ is captured by the action of $\mathscr{H}$ on $I(\tau)^{B}$. The irreducible representations of $\mathscr{H}$ have been classified ([K-L] and [G]). However, the decomposition of $I(\tau)^{B}$ itself, though well studied (see the references), is not completely understood. The purpose of this paper is to describe certain functions in $I(\tau)^{B}$ which are important for finding the submodules of this representation explicitly. This investigation enables us to extend some results of Rodier [R] and Rogawski [Ro], and also yields a new proof of the irreducibility criterion for $I(\tau)$ due to Kato and Müller ([Ka], [M]).

Recall (cf. [L]) that as a vector space, $\mathscr{H}$ is the tensor product of two subalgebras

$$
\mathscr{H}=\Theta \otimes \mathscr{H}_{W},
$$

where $\mathscr{H}_{W}$ is the Hecke algebra of the finite Weyl group $W$ of $G$ and $\Theta$ is isomorphic to the coordinate ring of a maximal torus $T$ in the complex Lie group which is dual to $G$ in the sense of Langlands. As a $\mathscr{H}_{W}$-module, $I(\tau)^{B}$ is always the regular representation of $\mathscr{H}_{W}$, so as $\tau$ varies, the change in the structure of $I(\tau)^{B}$ is seen in the action of 
$\Theta$. Now $\tau$ may be viewed as an element of $T$, hence as a character of $\Theta$, and the semisimplification of the $\Theta$-module $I(\tau)^{B}$ is isomorphic to $\bigoplus_{w \in W} \tau^{w}$. Moreover,

$$
\operatorname{Hom}_{G}\left(I(\tau), I\left(\tau^{w}\right)\right) \simeq \operatorname{Hom}_{\Theta}\left(I(\tau)^{B}, \tau^{w}\right) \simeq \operatorname{Hom}_{P}\left(I(\tau)_{N}, \tau^{w} \delta^{1 / 2}\right),
$$

where $I(\tau)_{N}$ is the Jacquet module with respect to the unipotent radical $N$ of $P$, and $\delta$ is the modulus of $P$.

We can write $I(\tau)^{B}$ as a direct sum of indecomposable $\Theta$-submodules, each containing a one-dimensional space of " $\Theta$-eigenvectors" which transform under $\Theta$ by the character $\tau^{w}$, for some $w \in W$. Moreover, any invariant subspace of $I(\tau)^{B}$ must contain a $\Theta$ eigenvector.

Our goal is to describe all $\Theta$-eigenvectors in $I(\tau)^{B}$ explicitly, and relate them to the structure of $I(\tau)^{B}$ as well as $\mathscr{H}$ itself. We are only partly successful. For some $\tau$, we have found all $\Theta$-eigenvectors. For arbitrary $\tau$, we have found all $\Theta$-eigenvectors for certain identifiable characters $\tau^{w}$. This is the content of Theorem (8.3), which is the culmination of this paper. In more detail, the contents are as follows.

We describe the $\Theta$-eigenvectors transforming by $\tau^{w}$ as linear combinations

$$
\sum_{y \in W} a_{w, y}(\tau) \varphi_{y}
$$

of standard basis elements $\varphi_{y} \in I(\tau)^{B}$, which correspond to $P \backslash G / B$ double cosets. Each $a_{w, y}$ is a rational function of $\tau$ (i.e., an element of the function field $\mathbb{C}(T)$ ) and is given by a recursive formula involving induction on the length of $y$. We have $a_{w, w}=1$ for all $w \in W$ and $a_{w, y}=0$ if $w \not \leq y$ in the Bruhat order. In the case that $\tau$ lies in no root kernel, Rogawski ([Ro]) has described all $\Theta$-eigenvectors in a completely different way, in terms of translates of a particular eigenvector by the Kazhdan-Lusztig basis of $\mathscr{H}_{W}$. Our improvement on this point is the determination of the basis coefficients $a_{w, y}$, and more importantly, their poles (see (5.2)). This gives us sharper information for singular $\tau$.

Actually, we give two recursive formulae for $a_{w, y}$ ((4.2) and (4.9)). The first comes from the compatibility of $\Theta$-eigenvectors with intertwining maps between principal series. The second formula arises from a relation in $\mathscr{H}$ between the generators of $\Theta$ and those of $\mathscr{H}_{W}$, which we use to study the action of $\mathscr{H}_{W}$ on the $\Theta$-eigenvectors. The formulae are in principle equivalent, of course, but it is not clear how 
to directly derive one from the other. Nevertheless, their similar appearance can be explained.

It is because intertwining operators come from multiplication in the "extended" Hecke algebra

$$
\widetilde{\mathscr{H}}:=\mathscr{H} \otimes_{\Theta} \mathbb{C}(T)=\mathscr{H}_{W} \otimes \mathbb{C}(T)
$$

by certain elements $\mathscr{T}_{w} \in \widetilde{\mathscr{H}}$. These were defined by Lusztig in [L] and are closely related to Rogawski's formulae for $\Theta$-eigenvectors. Consider the $\mathscr{H}$-submodule

$$
\widetilde{\mathscr{H}_{\tau}}=\mathscr{H} \otimes_{\Theta} \mathbb{C}(T)_{\tau^{w_{0}}}
$$

where $\mathbb{C}(T)_{\tau}$ consists of those functions which are holomorphic on $\tau \in T$, and $w_{0}$ is the long word in $W$. The $\mathscr{H}$-module $I(\tau)^{B}$ is a quotient of $\widetilde{\mathscr{H}}_{\tau}$. Assume for simplicity that no root of $T$ takes value $q^{ \pm 1}$ on $\tau$. For each $y \in W$, the usual (normalized) intertwining operator $\widetilde{\mathscr{A}_{y}} \in \operatorname{Hom}_{\mathscr{H}}\left(I(\tau)^{B}, I\left(\tau^{y}\right)^{B}\right)$ is the quotient of the map $\widetilde{\mathscr{H}}_{\tau} \rightarrow \widetilde{\mathscr{H}}_{\tau^{y}}$ given by right multiplication by $\mathscr{T}_{w_{0}^{-1} y w_{0}}$ (see (4.11)). Thus, both of our formulae for $a_{w, y}$ are related to multiplication by basis elements of $\widetilde{\mathscr{H}}$. Moreover, if $s \in W$ is a simple reflection, then $\mathscr{T}_{s}+1 \in\left(T_{s}+1\right) \Theta\left(T_{s}\right.$ is the generator of $\mathscr{H}_{W}$ corresponding to $\left.s\right)$, and this accounts for the similarity.

In $\S 5$, we digress to combine our point of view with that of [Ro] and derive an identity expressing the $a_{w, y}$ 's in terms of the product of certain Kazhdan-Lusztig basis elements of $\mathscr{H}_{W}$. A priori, this product involves Kazhdan-Lusztig polynomials, whose combinatorial complexity is much greater than that of our $a_{w, y}$ 's. In our identity, $\tau$ is playing the role of a deformation variable, and apparently some of the combinatorics is being expressed in terms of the $W$ action on the deformation space.

We return to our main path in $\S 6$ and find the poles of the $a_{w, y}$ 's. In principle, the recursive formulae should be enough for this, but other ideas were needed, namely the $P$-decomposition of $I(\tau)$ and the Jacquet module. In (6.2) we give a criterion for holomorphicity of $a_{w, y}$ which depends only on $w$, telling us on which root kernels our $\Theta$-eigenvectors are still defined.

If $\tau$ is not in the kernel of any root, then (and only then) the action of $\Theta$ on $I(\tau)^{B}$ is diagonalizable, all $a_{w, y}$ are holomorphic and we have found a basis of $I(\tau)^{B}$ consisting of $\Theta$-eigenvectors. As an application, we find, in (7.3), an explicit $\mathscr{H}$-composition series of $I(\tau)^{B}$ in the case that $\tau$ is regular. Consequently, we determine 
the lattice of submodules of $I(\tau)$ and generators of each submodule. This extends earlier work of Rodier ([R], see also [Ro]), in which the composition factors of $I(\tau)$ were parametrized.

If $\tau$ is unitary, we can use Harish-Chandra's theorem on intertwining operators to get a good upper bound on the number of $\Theta$ indecomposable summands in $I(\tau)^{B}$. From this we deduce that exactly the right number of $a_{w, y}$ 's are holomorphic at $\tau$, implying that we have found all of the $\Theta$-eigenvectors. This is the Hecke algebra version of the "Knapp-Stein dimension theorem" on intertwining operators, first proven by Silberger [S] in a more general context, and made explicit by Keyes [K]. We can then easily write down explicit spanning sets for the spaces of $\Theta$-eigenvectors in each isotypic component of $I(\tau)^{B}$.

The result for unitary $\tau$ is a special case of Theorem (8.3), which applies to at least one element, said to be "good", in the $W$ orbit of any $\tau$, and is a generalization of [Ro, Theorem (3.1)]. Assuming that $\tau$ itself is "good", we determine a non-empty set of $w \in W$, depending on the roots of $T$ which have (complex) modulus one on $\tau$, such that all $\Theta$-eigenvectors in $I(\tau)^{B}$ with character $\tau^{w}$ are linear combinations of the ones given by our formulae. Proceeding along Rogawski's lines, we can then strengthen Corollaries (3.2) and (3.3) of [Ro] as follows. In (8.6), we give weaker sufficient conditions, neither of which can be omitted, for $I(\tau)$ to have a unique irreducible quotient and submodule. In (8.7) we give a new proof of the irreducibility criterion for $I(t)^{B}$, originally announced in [M] and proven in [Ka].

In $\S 9$, the paper concludes with some examples and a conjecture related to the limitations of Theorem (8.3).

To make the paper easier to read, we have assumed that $G$ is split. This is not essential, and the motivated reader can adapt the computations to quasi-split groups.

I thank A. Moy, F. Shahidi and M.-F. Vigneras for useful discussions. Much of this work was done while I was provided with the stimulating environs of Universite de Paris VII and CIRM. Financial support was also received from NSA grant MDA904-89-H-2027.

1. Notation. Let $F$ be a nonarchimedean local field, and $\mathbf{G}$ a Chevalley group defined over $\mathscr{O}$, the ring of integers in $F$. We write $G$ for the $F$-rational points of $\mathbf{G}$, and use similar notation for other algebraic groups over $\mathscr{O}$.

Let $\mathbf{A}$ be a maximal $F$-split torus of $\mathbf{G}$, and denote the rational character group of $\mathbf{A}$ by $X^{*}(\mathbf{A})$. Let $\Delta, \Delta^{+}, \Delta^{-}, \Sigma \in X^{*}(\mathbf{A})$ be 
the roots of $\mathbf{A}$ in $\mathbf{G}$, a choice of positive roots, with corresponding negative and simple roots respectively. For $\alpha \in \Delta$, we let $h_{\alpha}: F^{\times} \rightarrow A$ be the corresponding one parameter subgroup. We set

$$
A^{-}=\left\{a \in A:|\alpha(a)|_{F} \leq 1 \forall \alpha \in \Sigma\right\} .
$$

Let $T=\mathbb{C}^{\times} \otimes X^{*}(\mathbf{A})$, the complex torus dual to $A$. Let $K=G(\mathscr{O})$, a maximal compact subgroup of $G$.

We have a pairing

$$
\langle,\rangle: A / A \cap K \times T \rightarrow \mathbb{C}^{\times}
$$

given by

$$
\langle a, z \otimes \lambda\rangle=z^{\operatorname{val}(\lambda(a))} .
$$

This induces an isomorphism between $A / A \cap K$ and the group $X^{*}(T)$ of rational characters of $T$ as well as an identification between $T$ and the group of unramified (quasi)-characters of $A$.

For $\alpha \in \Delta$ we define $e_{\alpha} \in X^{*}(T)$ by

$$
e_{\alpha}(\tau)=\left\langle h_{\alpha}(\varpi), \tau\right\rangle
$$

for $\tau \in T$.

The Weyl group $W=\operatorname{Norm}_{G}(A) / A$ acts on $A, X^{*}(A), T, X^{*}(T)$ and the characters of $A$ in such a way that

$$
\tau^{w}(a)=\tau\left(w a w^{-1}\right)
$$

and

$$
\left(w e_{\alpha}\right)(\tau)=e_{w \alpha}(\tau)=e_{\alpha}\left(\tau^{w}\right)
$$

for $w \in W, \tau \in T, a \in A, \alpha \in \Delta$. We set

$$
W_{\tau}=\left\{w \in W \mid \tau^{w}=\tau\right\}, \quad \Delta_{\tau}=\left\{\alpha \in \Delta \mid e_{\alpha}(\tau)=1\right\}, \quad \Delta_{\tau}^{+}=\Delta_{\tau} \cap \Delta^{+} .
$$

We also set

$$
T^{0}=\left\{\tau \in T \mid e_{\alpha}(\tau) \neq 1 \forall \alpha \in \Delta\right\}, \quad T^{00}=\left\{\tau \in T \mid W_{\tau}=1\right\} .
$$

If $w \in W$, there is a representative for $w$ in $K$ which we will also denote by $w$, hoping there is no confusion.

2. The affine Hecke algebra. Let $B \subset K$ be the Iwahori subgroup corresponding to our choice of simple roots $\Sigma$, and let $\mathscr{H}=$ $C_{c}^{\infty}(G / / B)$. As a vector space, $\mathscr{H}$ is the tensor product of two subalgebras

$$
\mathscr{H} \simeq \mathscr{H}_{W} \otimes \Theta
$$

which we now describe (see [L]). 
The subalgebra $\mathscr{H}_{W}$ consists of those functions in $\mathscr{H}$ which are supported on $K$. It is isomorphic to the finite dimensional Hecke algebra corresponding to $W$. The subalgebra $\Theta$ is commutative and has a linear basis $\left\{\theta_{a} \mid a \in A / A \cap K\right\}$ where the $\theta_{a}$ 's are defined as follows.

For $x \in G$, we let $T_{x} \in \mathscr{H}$ be the characteristic function of $B x B$. Let $a \in A$ and choose $a_{1}, a_{2} \in A^{-}$such that $a=a_{1} a_{2}^{-1}$. Then

$$
\theta_{a}=q^{\frac{1}{2}\left(l\left(a_{2}\right)-l\left(a_{1}\right)\right)} T_{a_{1}} T_{a_{2}}^{-1} .
$$

It is shown in [L] that this is independent of the choice of $a_{1}, a_{2}$, and then it is clear that $\theta_{a}$ depends only on the coset of $a$ in $A / A \cap K$. In this definition, $l$ denotes the length function on the normalizer in $G$ of $A \cap K$. It is defined by the equation $q^{l(x)}=[B x B: B]$. If $a \in A$, we have

$$
l(a)=\sum_{\alpha>0}|\operatorname{val}(\alpha(a))| .
$$

This means we can also write

$$
\theta_{a}=\delta^{1 / 2}(a) T_{a_{1}} T_{a_{2}}^{-1}
$$

where

$$
\delta(a)=\prod_{\alpha>0}|\alpha(a)|_{F}
$$

is the modulus of the Borel subgroup corresponding to our choice of simple roots.

Finally, we have the relation

$$
\theta_{a b}=\theta_{a} \theta_{b}, \quad a, b \in A .
$$

Thus $\Theta$ is isomorphic to the group algebra $\mathbb{C}[A / A \cap K]$. The pairing $\langle$,$\rangle from \S 1$ moreover induces a ring isomorphism

$$
\Theta \simeq \mathbb{C}[T]
$$

under which $\theta_{h_{\alpha}(\varpi)}$ corresponds to $e_{\alpha}$, and $\tau \in T$ is the algebra homomorphism $\tau: \Theta \rightarrow \mathbb{C}$ such that $\tau\left(\theta_{a}\right)=\langle a, \tau\rangle$.

Let $\mathbb{C}(T)$ be the field of rational functions on $T$. Note that $\mathbb{C}(T)$ is isomorphic to the quotient field of $\Theta$.

The Weyl group $W$ acts on $\mathbb{C}(T)$ by $(w \cdot a)(\tau)=a\left(\tau^{w}\right)$, for $w \in$ $W, \tau \in T$, and $a \in \mathbb{C}(T)$.

3. The unramified principal series. Let $P=A N$ be the Borel subgroup in $G$ whose unipotent radical $N$ is generated by root groups from $\Delta^{+}$. 
Our group $G$ has the double-coset decomposition

$$
G=\coprod_{w \in W} P w B
$$

According to [C, (1.3)], we have

$$
B w B \subseteq \bigcup_{x \geq w} P x P
$$

hence also

$$
P w B \subseteq \bigcup_{x \geq w} P x P
$$

Finally, we have

$$
P w B \cap P w P=(P \cap K) w(N \cap K) .
$$

For $\tau \in T$, we set

$$
I(\tau)=\operatorname{Ind}_{P}^{G}(\tau),
$$

where the induced representation consists of those locally constant functions $f$ on $G$ such that $f(p g)=\tau \delta^{1 / 2}(p) f(g)$, for $p \in P$, $g \in G$. The action is given by $[\pi(g) \varphi](h)=\varphi(h g)$, for $g, h \in G$ and $\varphi \in I(\tau)$. We sometimes suppress the $\tau$ and write simply $I$ instead of $I(\tau)$.

Since $\tau$ is unramified, we have, for each $w \in W$, a function $\varphi_{w}=$ $\varphi_{w}^{\tau} \in I(\tau)$ which is supported on $P w B$ and satisfies

$$
\varphi_{w}^{\tau}(p w b)=\tau \delta^{1 / 2}(p)
$$

for all $p \in P, b \in B$. The set of all $\varphi_{w}$ 's is a basis of the space $I(\tau)^{B}$ of Iwahori-invariant vectors in $I(\tau)$. Of course $I(\tau)^{B}$ affords a representation of $\mathscr{H}$. In particular, $I(\tau)^{B}$ affords an action of $\mathbb{C}[A / A \cap K]$ via $(a, \varphi) \mapsto \pi\left(\theta_{a}\right) \varphi, a \in A, \varphi \in I(\tau)^{B}$.

For any $P$-representation $(\pi, V)$, we denote the Jacquet module of $V$ by $\left(\pi_{N}, V_{N}\right)$.

(3.1) Proposition. The natural projection $j_{N}: I^{B} \rightarrow I_{N} \otimes \delta^{-1 / 2}$ is an isomorphism of $\mathbb{C}[A / A \cap K]$ modules.

Proof. That $j_{N}$ is a linear isomorphism is well known (cf. [C]). For the equivariance, we use $[\mathbf{C},(2.5)]$ which says

$$
j_{N}\left(\pi\left(T_{a}\right) \varphi\right)=[B a B: B] \pi_{N}(a) j_{N}(\varphi)
$$

for $a \in A^{-}, \varphi \in I$. We have therefore

$$
j_{N}(\varphi)=j_{N}\left(\pi\left(T_{a} T_{a}^{-1}\right) \varphi\right)=[B a B: B] \pi_{N}(a) j_{N}\left(\pi\left(T_{a}^{-1}\right) \varphi\right),
$$


from which we conclude

$$
j_{N}\left(\pi\left(T_{a}^{-1}\right) \varphi\right)=[B a B: B]^{-1} \pi_{N}\left(a^{-1}\right) j_{N}(\varphi) .
$$

Now let $a \in A$ be arbitrary, and write $a=a_{1} a_{2}^{-1}$ with $a_{i} \in A^{-}$. We get

$$
\begin{aligned}
j_{N}\left(\pi\left(\theta_{a}\right) \varphi\right) & =q^{\frac{1}{2}\left(l\left(a_{2}\right)-l\left(a_{1}\right)\right)} \frac{\left[B a_{1} B: B\right]}{\left[B a_{2} B: B\right]} \pi_{N}(a) j_{N}(\varphi) \\
& =q^{\frac{1}{2}\left(l\left(a_{1}\right)-l\left(a_{2}\right)\right)} \pi_{N}(a) j_{N}(\varphi) \\
& =\delta^{-\frac{1}{2}}(a) \pi_{N}(a) j_{N}(\varphi) .
\end{aligned}
$$

In other words, the $\Theta$-module structure of $I^{B}$ is essentially the same as the $A$-module structure of $I_{N}$. This relationship can be strengthened as follows.

There is a $P$-stable filtration $I=\bigcup_{w \in W} I_{w}$, where

$$
I_{w}=I(\tau)_{w}=\left\{f \in I(\tau) \mid \text { support of } f \subseteq \bigcup_{x \geq w} P x P\right\} .
$$

This gives an $A$-filtration

$$
I_{N}=\bigcup_{w \in W} I_{w, N}
$$

hence a $\Theta$-filtration

$$
I^{B}=\bigcup_{w \in W} I_{w} \cap I^{B} .
$$

Observe that $x \geq w \Rightarrow \varphi_{x} \in I_{w} \cap I^{B}$.

(3.2) Proposition. The projection $j_{N}$ induces an isomorphism $I_{w} \cap I^{B} \cong I_{w, N}$. Moreover, $\left\{\varphi_{x} \mid x \geq w\right\}$ is a basis of $I_{w} \cap I^{B}$.

Proof. By [C, (2.3)], the quotient

$$
I_{w, N} / \sum_{x>w} I_{x, N}
$$

is one-dimensional, so

$$
\operatorname{dim} I_{w, N}=\sharp\{x \in W \mid x \geq w\} .
$$

On the other hand, counting $\varphi_{x}$ 's shows

$$
\operatorname{dim} I_{w} \cap I^{B} \geq \sharp\{x \in W \mid x \geq w\} .
$$

This completes the proof, since $j_{N}$ is injective. 
For the rest of this section, we assume $\tau \in T^{00}$, and recall some facts about intertwining operators that we will need later. See [C].

For $x \in W$, let $N_{x}$ be the subgroup of $N$ generated by the roots in

$$
R(x):=\{\alpha>0 \mid x \alpha<0\} .
$$

Since $\tau \in T^{00}$, the space $\operatorname{Hom}_{G}\left(I(\tau), I\left(\tau^{x}\right)\right)$ is one dimensional, spanned by an operator $\mathscr{A}_{x}=\mathscr{A}_{x}^{\tau}$ with the property that

$$
\mathscr{A}_{x}(f)(1)=\int_{N_{x}} f(x n) d n,
$$

if the support of $f$ lies in

$$
\bigcup_{y \nless x} P y P .
$$

(The measure on $N_{x}$ is such that $\operatorname{vol}\left(N_{x} \cap K\right)=1$.) Finally, if $x, y \in W$ satisfy $l(x)+l(y)=l(x y)$ then $\mathscr{A}_{y}^{\tau^{x}} \mathscr{A}_{x}^{\tau}=\mathscr{A}_{x y}^{\tau}$.

4. $\Theta$-eigenvectors. We begin with a basic property of certain $\Theta$ eigenvectors.

(4.1) LEMMA. Let $\tau \in T$, and assume $w \in W$ is maximal in its coset $W_{\tau} w$, with respect to the Bruhat order on $W$. Then there exists a unique function $f_{w}^{\tau} \in I(\tau)_{w} \cap I(\tau)^{B}$ such that

(1) $f_{w}^{\tau}(w)=1$, and

(2) $\pi\left(\theta_{a}\right) f_{w}^{\tau}=\tau^{w}(a) f_{w}^{\tau}$ for all $a \in A$.

Proof. In [C] it is shown, for any $x \in W$, that $A$ acts on the one dimensional quotient

$$
I_{x, N} / \sum_{y>x} I_{y, N}
$$

by the character $\tau^{x} \delta^{\frac{1}{2}}$. By (3.2), $\Theta$ acts on the quotient

$$
I_{x} \cap I^{B} / \sum_{y>z} I_{y} \cap I^{B}
$$

by the character $\tau^{x}$.

Our hypothesis on $w$ implies that $\tau^{w}$ does not appear in any $\Theta$ invariant subquotient of $\sum_{y>w} I_{y} \cap I^{B}$. Hence the quotient affording $\tau^{w}$ splits off and we can find a nonzero $f_{w}^{\tau}$ as in (2). Moreover, $f_{w}^{\tau}$ must have $P w B$ in its support, so we can normalize to get (1). 
For $\alpha \in \Delta$, we set

$$
c_{\alpha}=\frac{1-q^{-1} e_{\alpha}}{1-e_{\alpha}} \in \mathbb{C}(T) .
$$

If we have also an element $w \in W$, and $\alpha>0$, we define

$$
K_{\alpha, w}= \begin{cases}c_{\alpha} c_{-\alpha} & \text { if } w^{-1} \alpha>0 \\ 1 & \text { if } w^{-1} \alpha<0\end{cases}
$$

If $\alpha<0$, we set $K_{\alpha, w}=K_{-\alpha, w}$.

Thus, $K_{w, \alpha}$ is an element of $\mathbb{C}(T)$ which is holomorphic on $T^{0}$.

(4.2) Theorem. Let $\tau \in T^{00}, w \in W$, and $\alpha \in \Sigma$. Set $s=s_{\alpha}$. Then (1) $\mathscr{A}_{s} f_{w}^{\tau}=K_{\alpha, w}(\tau) f_{s w}^{\tau^{s}}$

$$
f_{w}^{\tau}=\sum_{y \geq w} a_{w, y}(\tau) \varphi_{y}^{\tau}
$$

where the $a_{x, y} \in \mathbb{C}(T)$ are given recursively as follows:

$$
\begin{array}{ll}
a_{x, y}=0 & \text { if } x \not \leq y, \\
a_{x, x}=1 & \text { for all } x \in W
\end{array}
$$

and finally, if $s y>y$, then

$$
a_{x, s y}=\left(1-c_{\alpha}\right) a_{x, y}+K_{\alpha, x}\left(s \cdot a_{s x, y}\right) .
$$

Proof. For any $x \in W$ and $\tau_{1} \in T^{00}$, we can write

$$
f_{x}^{\tau_{1}}=\sum_{y \in W} a_{x, y}\left(\tau_{1}\right) \varphi_{y}^{\tau_{1}}
$$

for some complex numbers $a_{x, y}\left(\tau_{1}\right)$. By definition (see (4.1)), we have $a_{x, x}\left(\tau_{1}\right)=1$ and $a_{x, y}\left(\tau_{1}\right)=0$ if $x \not \leq y$. We set $a_{y}=a_{w, y}(\tau)$, $b_{y}=a_{s w, y}\left(\tau^{s}\right)$.

Note that $\mathscr{A}_{s} f_{w}^{\tau}$ and $f_{s w}^{\tau^{s}}$ are two functions in $I\left(\tau^{s}\right)^{B}$ which transform by $\tau^{w}$ under $\Theta$. The regularity of $\tau$ implies that this character has multiplicity one, so there is a $k=k_{\alpha}, w, \tau \in \mathbb{C}$ such that

$$
\mathscr{A}_{s} f_{w}^{\tau}=k f_{s w}^{\tau^{s}} \text {. }
$$

From [C, (3.4)], we have

$$
\begin{array}{ll}
\mathscr{A}_{s} \varphi_{y}^{\tau}=\left(c_{\alpha}(\tau)-1\right) \varphi_{y}^{\tau^{s}}+q^{-1} \varphi_{s y}^{\tau^{s}} & \text { if } s y>y, \\
\mathscr{A}_{s} \varphi_{y}^{\tau}=\left(c_{\alpha}(\tau)-q^{-1}\right) \varphi_{y}^{\tau^{s}}+\varphi_{s y}^{\tau^{s}} & \text { if } s y<y,
\end{array}
$$


So

$$
\begin{aligned}
k \sum_{y} b_{y} \varphi_{y}^{\tau^{s}}= & \sum_{s y>y} a_{y}\left[\left(c_{\alpha}(t)-1\right) \varphi_{y}^{\tau^{s}}+q^{-1} \varphi_{s y}^{\tau^{s}}\right] \\
& +\sum_{s y<y} a_{y}\left[\left(c_{\alpha}(t)-q^{-1}\right) \varphi_{y}^{\tau^{s}}+\varphi_{s y}^{\tau^{s}}\right]
\end{aligned}
$$

Comparing coefficients of $\varphi_{z}^{\tau^{s}}$, we find

$$
k b_{z}= \begin{cases}\left(c_{\alpha}(\tau)-1\right) a_{z}+a_{s z} & \text { if } s z>z, \\ \left(c_{\alpha}(\tau)-q^{-1}\right) a_{z}+q^{-1} a_{s z}, & \text { if } s z<z .\end{cases}
$$

In particular,

$$
k b_{s w}= \begin{cases}\left(c_{\alpha}(\tau)-1\right) a_{s w}+a_{w} & \text { if } s w<w \\ \left(c_{\alpha}(\tau)-q^{-1}\right) a_{s w}+q^{-1} a_{w} & \text { if } s w>w .\end{cases}
$$

But $b_{s w}=a_{w}=1$, and $a_{s w}=0$ if $s w<w$. We also have

(4.3) LemmA. $a_{s w}=1-c_{\alpha}(\tau)$ if $s w>w$.

We first prove

(4.4) LEMMA.

(1) $\mathscr{A}_{x} \varphi_{w}(1) \neq 0 \Rightarrow w \leq x$.

(2) $\mathscr{A}_{w} \varphi_{w}(1)=1$

(3) $\mathscr{A}_{s w} \varphi_{w}(1)=c_{\alpha}(\tau)-1$ if $w \leq s w$.

Proof. Assume $w \not \leq x$. Then

$$
P w B \subseteq \bigcup_{y \geq w} P y P \subseteq \bigcup_{y \notin x} P y P,
$$

so

$$
\mathscr{A}_{x} \varphi_{w}(1)=\int_{N_{x}} \varphi_{w}(x n) d n .
$$

However, if $x N$ meets $P w B$ then $x N$ meets $P y P$, for some $y \geq w$, which must in fact equal $x$, and this is a contradiction.

For (2), we start with the fact that $P w P \cap B w B=(P \cap K) w(N \cap K)$. Thus if $w n \in p w B$ for some $n \in N_{w}, p \in P$, we have $p^{-1} w n \in$ $(P \cap K) w(N \cap K)$, so $w n \in P w(N \cap K)$. By uniqueness of expression 
in the Bruhat decomposition, we have $n \in N \cap K$, and then also $p \in$ $P \cap K$. Moreover this shows, since $\tau$ is unramified, that $\varphi_{w}(w n)=1$. We now can compute the integral:

$$
\begin{aligned}
\mathscr{A}_{w} \varphi_{w}(1) & =\int_{N_{w}} \varphi_{w}(w n) d n \\
& =\int_{N_{w} \cap K} \varphi_{w}(w n) d n=\int_{N_{w} \cap K} d n=1 .
\end{aligned}
$$

For (3), we use the cocycle condition for the operators $\mathscr{A}_{x}$ to get

$$
\begin{aligned}
\mathscr{A}_{s w} \varphi_{w}(1) & =\mathscr{A}_{w} \mathscr{A}_{s} \varphi_{w}(1) \\
& =\left(c_{\alpha}(\tau)-1\right) \mathscr{A}_{w} \varphi_{w}^{\tau^{s}}(1)+q^{-1} \mathscr{A}_{w} \varphi_{s w}^{\tau^{s}}(1) \\
& =c_{\alpha}(\tau)-1
\end{aligned}
$$

by (1) and (2).

We are now able to give the

Proof of Lemma (4.3). The functional $f \mapsto \mathscr{A}_{\text {sw }} f(1)$ on $I(\tau)$ transforms under $A$ by the character $\tau^{s w} \delta^{\frac{1}{2}}$, so $\mathscr{A}_{s w} f_{w}^{\tau}(1)=0$. On the other hand, (4.4) implies

$$
\mathscr{A}_{s w} f_{w}^{\tau}(1)=a_{s w}+a_{w} \mathscr{A}_{s w} \varphi_{w}(1)=a_{s w}+c_{\alpha}(\tau)-1 .
$$

Now, using the easily checked identity $\left(c_{\alpha}-q^{-1}\right)\left(1-c_{\alpha}\right)+q^{-1}=$ $c_{\alpha} c_{-\alpha}$, we see that $k=K_{\alpha, w}(\tau)$, and this proves (1) in (4.2). We have also shown that

$$
a_{w, s y}(\tau)=\left(c_{\alpha}(\tau)-1\right) a_{w, y}(\tau)+K_{\alpha, w}(\tau) a_{s w, y}\left(\tau^{s}\right) \quad \text { if } s y>y,
$$

so that we have finished the proof of (4.2).

(4.5) Corollary. Let $t \in T^{00}, y, w \in W$. Then

$$
\mathscr{A}_{y}\left(f_{w}^{\tau}\right)=\prod_{R\left(y^{-1}\right)} K_{\alpha, w}(\tau) f_{y^{-1} w^{y}}^{\tau^{y}}
$$

(Recall that $\left.R\left(y^{-1}\right)=\left\{\alpha>0: y^{-1} \alpha>0\right\}.\right)$

Proof. Suppose the result is true for $y$ and let $s$ be a simple reflection such that $y s>y$. Then

$$
\begin{aligned}
\mathscr{A}_{y s} f_{w}^{\tau} & =\mathscr{A}_{S} \prod_{\beta \in R\left(y^{-1}\right)} K_{\beta, w}(\tau) f_{y^{-1} w}^{\tau^{y}} \\
& =\prod_{R\left(y^{-1}\right)} K_{\beta, w}(\tau) K_{\alpha, y^{-1} w}\left(\tau^{y}\right) f_{s y^{-1} w}^{\tau^{y s}} .
\end{aligned}
$$


Since $R\left(s y^{-1}\right)=R\left(y^{-1}\right) \cup\{y \alpha\}$ and $K_{\alpha, y^{-1} w}\left(\tau^{y}\right)=K_{y \alpha, w}(\tau)$, we have the result by induction.

We can view the $f_{w}^{\tau}$ 's as specializations of elements in $\widetilde{\mathscr{H}}:=\mathbb{C}(T) \otimes$ $\mathscr{H}_{W}=\mathbb{C}(T) \otimes_{\Theta} \mathscr{H}$ as follows. Define

$$
f_{w}=\sum_{y \geq w} a_{w, y} T_{y} \in \widetilde{\mathscr{H}}
$$

Let $\mathscr{P}_{\tau}: C_{c}^{\infty}(G) \rightarrow I(\tau)$ be the projection given by

$$
\mathscr{P}_{\tau}(f)(g)=\int_{P} \tau^{-1} \delta^{-\frac{1}{2}}(p) f(p g) d p,
$$

where the measure on $P$ is such that the volume of $P \cap K=1$. For $w \in W$, we have $\mathscr{P}_{\tau}\left(T_{w}\right)=\varphi_{w}^{\tau}$.

We let $\mathbb{C}(T)_{\tau}$ denote the ring of functions in $\mathbb{C}(T)$ which are holomorphic at $\tau$. We have a homomorphism of right $\mathscr{H}_{W}$-modules

$$
\mu_{\tau}: \mathbb{C}(T)_{\tau} \otimes \mathscr{H}_{W} \rightarrow \mathscr{H}_{W}
$$

given by evaluation of the coefficients at $\tau$. If $\tau \in T^{0}$ then all $a_{w, y}$ 's belong to $\mathbb{C}(T)_{\tau}$ and

$$
f_{w}^{\tau}=\mathscr{P}_{\tau}\left(\mu_{\tau}\left(f_{w}\right)\right) .
$$

Since $a_{w, y}=0$ if $w \not \leq y$ and $a_{w, w}=1$, the $f_{w}$ 's form a basis of the $\mathbb{C}(T)$-vector space $\widetilde{\mathscr{H}}$.

We next describe the effect of right multiplication by a standard generator $T_{s}$ in terms of this basis. Applying $\mathscr{P}_{\tau} \circ \mu_{\tau}$ to this relation tells how $T_{S}$ acts on the $\Theta$-eigenvectors. In the process, we will derive another recursive formula for the $a_{x, y}$ 's.

First of all, an easy computation shows, for $y$ and $w \in W$, that

$$
\pi\left(T_{y}\right) \mathscr{P}_{\tau}\left(T_{w}\right)=\mathscr{P}_{\tau}\left(T_{w} T_{y^{-1}}\right) .
$$

Thus, if $s$ is a simple reflection in $W$, we have

$$
\pi\left(T_{s}\right) \varphi_{w}= \begin{cases}\varphi_{w s} & \text { if } w s>w, \\ q \varphi_{w s}+(q-1) \varphi_{w} & \text { if } w s<w .\end{cases}
$$

The following relation in $\mathscr{H}$ is fundamental $([\mathbf{L},(3.9)])$. Let $s=$ $s_{\alpha}, \alpha \in \Sigma$, and let $a \in A$. Set $\theta_{\alpha}=\theta_{h_{\alpha}(\varpi)}$. Then

$$
\theta_{a}\left(T_{s}+1\right)-\left(T_{s}+1\right) \theta_{\text {sas }^{-1}}=\left(\theta_{a}-\theta_{\text {sas }^{-1}}\right) \frac{1-q \theta_{\alpha}}{1-\theta_{\alpha}} .
$$

The right-hand side is actually in $\mathscr{H}$. 
(4.8) Lemma. Let $M$ be any $\mathscr{H}$-module. Suppose that $f \in M$ is an eigenvector for $\Theta$ with character $\tau$, and $e_{\alpha}(\tau) \neq 1$. Then $\left(\pi\left(T_{s}\right)+q c_{\alpha}(\tau)-q\right) f$ is a $\Theta$-eigenvector in $M$ with character $\tau^{s}$.

Proof. We have

$$
\pi\left(\theta_{\alpha}\right) f=\tau\left(h_{\alpha}(\varpi)\right) f=e_{\alpha}(\tau) f,
$$

so the fundamental relation gives

$$
\pi\left(\theta_{a}\left(T_{s}+1\right)\right) f \tau\left(s a s^{-1}\right) \pi\left(T_{s}+1\right) f+q c_{\alpha}(\tau)\left(\tau(a)-\tau\left(s a s^{-1}\right)\right) f .
$$

Since $e_{\alpha}(\tau) \neq 1$, this can be rewritten as

$$
\pi\left(\theta_{a}\right)\left[\pi\left(T_{s}\right) f+q\left(c_{\alpha}(\tau)-1\right) f\right]=\tau\left(\operatorname{sas}^{-1}\right)\left[\pi\left(T_{s}\right) f+q\left(c_{\alpha}(\tau)-1\right) f\right],
$$

whence the claim.

We introduce another bit of notation: For $\alpha \in \Delta^{+}, w \in W$, set

$$
J_{\alpha, w}= \begin{cases}c_{w \alpha} c_{-w \alpha} & \text { if } w \alpha>0 \\ 1 & \text { if } w \alpha<0\end{cases}
$$

Note that $J_{\alpha, w}(\tau)=K_{\alpha, w^{-1}}\left(\tau^{w}\right)$.

(4.9) Proposition. Let $w \in W, \alpha \in \Sigma, s=s_{\alpha}$. Then

(1) $f_{w} T_{s}=q\left(1-c_{w \alpha}\right) f_{w}+q J_{\alpha, w} f_{w s}$, in $\widetilde{\mathscr{H}}$.

(2) Suppose $y \in W$ and $y s>y$. Then

$$
a_{w, y s}=\left(1-c_{w \alpha}\right) a_{w, y}+J_{\alpha, w} a_{w s, y} .
$$

Proof. We show that (1) and (2) hold when both sides are evaluated at an arbitrary $\tau \in T^{00}$. Then we will use (2) to get (1) as stated above.

Since, for $\tau \in T^{00}$, the algebra $\Theta$ is diagonalizable on $I(\tau)^{B}$ with distinct eigencharacters, it follows from (4.8) that

$$
\pi\left(T_{s}\right) f_{w}^{\tau}+q\left(c_{w \alpha}(\tau)-1\right) f_{w}^{\tau}=C f_{w s}^{\tau}
$$

for some complex number $C=C_{\alpha, w, \tau}$. We will compute this constant in the process of finding the new recursive formula for $a_{x, y}$. We have

$$
\begin{aligned}
C \sum_{y} a_{w s, y} \varphi_{y}^{\tau}= & C f_{w s}^{\tau}=\pi\left(T_{s}\right) f_{w}^{\tau}+q\left(c_{w \alpha}(\tau)-1\right) f_{w}^{\tau} \\
= & \sum_{y s>y} a_{w, y} \varphi_{y s}+\sum_{y s<y} a_{w, y}\left(q \varphi_{y s}+(q-1) \varphi_{y}\right) \\
& +q\left(c_{w \alpha}(\tau)-1\right) \sum_{y} a_{w, y} \varphi_{y} .
\end{aligned}
$$


Comparing coefficients leads to the formula

$$
C a_{w s, y}= \begin{cases}q a_{w, y s}+q\left(c_{w \alpha}(\tau)-1\right) a_{w, y} & \text { if } y s>y, \\ a_{w, y s}+q\left(c_{w \alpha}(\tau)-q^{-1}\right) a_{w, y} & \text { if } y s<y .\end{cases}
$$

Letting $y=w s$, we get

$$
C= \begin{cases}q & \text { if } w s<w \\ 1+\left(q c_{w \alpha}(\tau)-1\right) a_{w, w s} & \text { if } w s>w .\end{cases}
$$

Suppose $w s>w$, and let $w^{\prime}=w s$. Replacing $w$ by $w^{\prime}$, so that we know $C_{\alpha, w^{\prime}, \tau}=q$, we get

$$
q a_{w, w s}=q a_{w^{\prime} s, w^{\prime}}=q c_{w^{\prime} \alpha}(\tau)-1=q\left(c_{w s \alpha}-q^{-1}\right)=q\left(1-c_{w \alpha}\right) .
$$

This means $C=1+q\left(c_{w \alpha}-q^{-1}\right)\left(1-c_{w \alpha}\right)$, and this expression equals $q c_{w \alpha} c_{-w \alpha}$. Thus, if $y s>y$, we have

$$
q a_{w, y s}=q\left(1-c_{w \alpha}\right) a_{w, y}+C a_{w s, y}=q\left(1-c_{w \alpha}\right) a_{w, y}+q J_{\alpha, w} a_{w s, y} .
$$

This proves (2).

To prove (1), we must show that

$$
\begin{aligned}
& \sum_{y s>y} a_{w, y} T_{y s}+\sum_{y s<y} a_{w, y}\left(q T_{y s}+(q-1) T_{y}\right) \\
& \quad=q\left(1-c_{w \alpha}\right) \sum_{y \in W} a_{w, y} T_{y}+q J_{\alpha, w} \sum_{y \in W} a_{w s, y} T_{y} .
\end{aligned}
$$

We compare coefficients of $T_{y}$ on both sides. If $y s>y$, we must show that

$$
q a_{w, y s}=q\left(1-c_{w \alpha}\right) a_{w, y}+q J_{\alpha, w} a_{w s, y},
$$

but this is just (2), which was already shown. If $y s<y$, the equation to be verified is

$$
a_{w, y s}+a_{w, y}(q-1)=q\left(1-c_{w \alpha}\right) a_{w, y}+q J_{\alpha, w} a_{w s, y} .
$$

We rewrite this as

$$
q\left(q^{-1}-c_{w \alpha}\right) a_{w, y}+q J_{\alpha, w} a_{w s, y}=a_{w, y s} .
$$

Now apply (2), with $y$ replaced by $y s$, to both terms on the left of this last expression, and use the relation

$$
\left(q^{-1}-c_{w \alpha}\right)\left(1-c_{w \alpha}\right)=q^{-1}-c_{w \alpha} c_{-w \alpha} .
$$

We get

$$
\begin{aligned}
q\left(q^{-1}-c_{w \alpha}\right) a_{w, y}= & q\left(q^{-1}-c_{w \alpha} c_{-w \alpha}\right) a_{w, y s} \\
& +q J_{\alpha, w}\left(q^{-1}-c_{w \alpha}\right) a_{w s, y s}
\end{aligned}
$$


and

$$
q J_{\alpha, w} a_{w s, y}=q J_{\alpha, w}\left[\left(c_{w \alpha}-q^{-1}\right) a_{w s, y s}+J_{\alpha, w s} a_{w, y s}\right] .
$$

Now we add the two expressions and observe that $J_{\alpha, w} J_{\alpha, w s}=$ $c_{w \alpha} c_{-w \alpha}$. This gives (1).

(4.10) Corollary. For $w, y \in W$, we have

$$
f_{w} T_{y}=q^{l(y)}\left(\prod_{\alpha \in R(y)} J_{\alpha, w}\right) f_{w y}+\sum_{z<y} c_{z} f_{w z}
$$

for certain $c_{z} \in \mathbb{C}(T)$.

Proof. This follows easily by induction on the length of $y$, just as in (4.5).

One reason for the similarity between the formulae in $(4.2)(2)$ and (4.9)(2) is that the intertwining operators $\mathscr{A}_{S}$ are also specializations of multiplication operators in $\widetilde{\mathscr{H}}:=\mathscr{H} \otimes_{\Theta} \mathbb{C}(T)$. By this we mean the following. Consider the left $\mathscr{H}$-module

$$
\widetilde{\mathscr{H}_{\tau}}=\mathscr{H} \otimes_{\Theta} \mathbb{C}(T)_{\tau^{w_{0}}},
$$

where $\Theta$ is viewed as a subring of $\mathbb{C}(T)_{\tau} w_{0}$. We have a surjection of left $\mathscr{H}$-modules

$$
\sigma_{\tau}: \widetilde{\mathscr{H}}_{\tau} \rightarrow I(\tau)^{B}
$$

given by $\sigma_{\tau}(T \otimes f)=f\left(\tau^{w_{0}}\right) \pi(T) f_{w_{0}}^{\tau}$, for $T \in \mathscr{H}$ and $f \in \mathbb{C}(T)_{\tau^{w_{0}}}$.

Let $s=s_{\alpha}$ be a simple reflection. If $e_{\alpha}(\tau) \neq 1$, the intertwining map $\mathscr{A}_{S}: I(\tau)^{B} \rightarrow I\left(\tau^{S}\right)^{B}$ is defined $([\mathbf{K}])$. If $e_{\alpha}(\tau)=1$, the normalized intertwining map $\widetilde{\mathscr{A}}_{S}=\frac{1}{c_{\alpha}} \mathscr{A}_{S} \in \operatorname{End}_{\mathscr{H}}\left(I(\tau)^{B}\right)$ is defined.

On the other hand, Luzstig ([L]) has introduced another basis $\left\{\mathscr{T}_{w}: w \in W\right\}$ of $\widetilde{\mathscr{H}}$ consisting of invertible elements satisfying $\mathscr{T}_{x} \mathscr{T}_{y}$ $=\mathscr{T}_{x y}$ for all $x, y \in W$. It suffices to know that

$$
\mathscr{T}_{s}=\frac{1}{c_{\alpha}}\left[q^{-1} T_{s}+c_{\alpha}-1\right] \text {. }
$$

Set $\bar{\alpha}=-w_{0} \alpha \in \Sigma$, and $\bar{s}=s_{\bar{\alpha}}$. If $e_{\alpha}(\tau) \neq 1$, we have $\widetilde{\mathscr{H}}_{\tau} c_{\bar{\alpha}} \mathscr{T}_{\bar{s}}=$ $\widetilde{\mathscr{H}}_{\tau^{s}}$, and if $e_{\alpha}(\tau)=1$ then $\widetilde{\mathscr{H}}_{\tau} \mathscr{T}_{\bar{s}}=\widetilde{\mathscr{H}}_{\tau}$.

(4.11) Proposition. For all $T \in \widetilde{\mathscr{H}}_{\tau}$ we have

$$
\begin{aligned}
& \mathscr{A}_{s} \sigma_{\tau}(T)=\sigma_{\tau^{s}}\left(T c_{\bar{\alpha}} \mathscr{T}\right), \quad \text { if } e_{\alpha}(\tau) \neq 1, \quad \text { and } \\
& \widetilde{\mathscr{A}_{s}} \sigma_{\tau}(T)=\sigma_{\tau}(T \mathscr{\mathscr { T }}), \quad \text { if } e_{\alpha}(\tau)=1 \text {. }
\end{aligned}
$$


Proof. It is enough to check the equations when $T=T_{e}$ (= the identity of $\widetilde{\mathscr{H}})$. In the first case, we need only then verify that

$$
\mathscr{A}_{s}\left(\varphi_{w_{0}}^{\tau}\right)=q^{-1} \pi\left(T_{\bar{s}}\right) \varphi_{w_{0}}^{\tau^{s}}+c_{\bar{\alpha}}\left(\tau^{s w_{0}}\right)-1 .
$$

The right-hand side is

$$
\varphi_{s w_{0}}^{\tau^{s}}+\left(c_{\alpha}(\tau)-q^{-1}\right) \varphi_{w_{0}}^{\tau^{s}}
$$

which is nothing but the formula for $\mathscr{A}_{s}\left(\varphi_{w_{0}}^{\tau}\right)$ for $\tau \in T^{00}$ (see proof of (4.2)). Both sides of the formula are holomorphic in a neighborhood of $e_{\alpha}=1$, so it is valid throughout that domain.

A similar argument applies to $\widetilde{\mathscr{A}_{S}}$ in a neighborhood of $e_{\alpha}=1$.

5. Some identities. We make in this section some observations about the $a_{w, y}(\tau)$ 's for particular values of $\tau \in T^{0}$. We need some notation: If $J \subseteq \Sigma$, then $W_{J}$ is the subgroup of $W$ generated by the roots in $J$ and $W^{J}=\left\{w \in W: w^{-1} J \subseteq \Delta^{+}\right\}$.

(4.12) Proposition. (1) Let $J \subseteq\left\{\alpha \in \Sigma: e_{\alpha}(\tau)=q\right\}, x \in W^{J}$. Then

$$
a_{x, z y}(\tau)=a_{x, y}(\tau) \text { for all } z \in W_{J}, y \in W^{J} .
$$

(2) Let $L \subseteq\left\{\alpha \in \Sigma: e_{\alpha}(\tau)=q^{-1}\right\}, x \in W^{L}$. Then

$$
a_{x, z y}(\tau)=(-q)^{-l(z)} a_{x, y}(\tau) \text { for all } z \in W_{L}, y \in W^{L} .
$$

Proof. We prove (2) by induction on the length of $z$; the proof of (1) is the same. If $\alpha \in L$, and $s_{\alpha} z>z$, then $s_{\alpha} z y>z y$. Also, $K_{\alpha, w}(\tau)=0$ so

$$
a_{x, s_{\alpha} z y}(\tau)=\left(1-c_{\alpha}(\tau)\right) a_{x, z y}(\tau)=\left(-q^{-1}\right) a_{x, z y}(\tau) .
$$

The formula in (2) follows immediately.

For example, if $\left\{\alpha \in \Sigma: e_{\alpha}(\tau)=q^{-1}\right\}=\Sigma$, then

$$
f_{e}^{\tau}=\sum_{w \in W}(-q)^{-l(w)} \varphi_{w}^{\tau}
$$

spans the Iwahori invariants in the Steinberg representation. If the above set of roots is instead equal to $-\Sigma$, we get that $f_{e}^{\tau}=\sum_{w \in W} \varphi_{w}^{\tau}$ is the spherical vector in $I(\tau)$. These seem to be the only cases where it is possible to compute $f_{w}^{\tau}$ in closed form for a general group.

We now record an identity between the $a_{w, y}$ 's and the KazhdanLusztig basis $\left\{C_{w}: w \in W\right\}$ of $\mathscr{H}_{W}$. For the complete definition of this basis, see [K-L1]. We mention only that $C_{s}=q^{\frac{1}{2}}\left(q^{-1} T_{s}-1\right)$ for 
a simple reflection $s$, that if $w s<w$ then $C_{w} C_{s}=-\left(q^{\frac{1}{2}}+q^{-\frac{1}{2}}\right) C_{w}$, and that if $w s>w$, we have

$$
C_{w} C_{s}=C_{w s}+\sum_{z s<z} \mu(z, w) C_{z}
$$

where $\mu(z, w)$ is the coefficient of $u^{\frac{1}{2}(l(w)-l(z)-1)}$ in the polynomial $P_{z, w}(u)$ defined in $[\mathbf{K}-\mathbf{L} \mathbf{1}]$.

(5.2) Proposition. Let $\tau \in T^{0}$ and let $w=s_{1} \cdots s_{r}$ be a reduced expression for $w \in W$, where each $s_{i}$ is the simple reflection for some $\alpha_{i} \in \Sigma$. Then

$$
\left[C_{S_{1}}+q^{\frac{1}{2}} c_{\alpha_{1}}(\tau)\right] \cdots\left[C_{S_{r}}+q^{\frac{1}{2}} c_{\alpha_{r}}(\tau)\right]=q^{\frac{r}{2}} \sum_{y \geq w_{0} w} a_{w_{0} w, y}(\tau) T_{w_{0}}^{-1} T_{y} .
$$

In particular, if $\tau_{0} \in T$ is such that the Steinberg module is the irreducible quotient of $I\left(\tau_{0}\right)$ then

$$
C_{S_{1}} \cdots C_{s_{r}}=q^{\frac{r}{2}} \sum_{y \geq w_{0} w} a_{w_{0} w, y}\left(\tau_{0}\right) T_{w_{0}}^{-1} T_{y}
$$

Proof. Let $s=s_{\alpha}$ be a simple reflection. As in [Ro], we consider the element

$$
q^{-\frac{1}{2}} C_{S}+c_{\alpha}(\tau)=q^{-1} T_{S}+c_{\alpha}(\tau)-1 \in \mathscr{H}_{W} .
$$

If $w s<w$ we showed in the proof of (4.9) that

$$
\pi\left(q^{-\frac{1}{2}} C_{s}+c_{\alpha}(\tau)\right) f_{w}^{\tau}=f_{w s}^{\tau},
$$

from which it follows that

$$
\pi\left(\left[C_{S_{r}}+q^{\frac{1}{2}} c_{\alpha_{r}}(\tau)\right] \cdots\left[C_{S_{1}}+q^{\frac{1}{2}} c_{\alpha_{1}}(\tau)\right]\right) f_{w_{0}}^{\tau}=q^{\frac{r}{2}} f_{w_{0} w}^{\tau} \cdot
$$

Let $\sigma: \mathscr{H}_{W} \rightarrow I(\tau)^{B}$ be the linear isomorphism sending $T_{x}$ to $\varphi_{x}$ for all $s \in W$. By our remarks prior to (4.8) we have

$$
\sigma\left(T_{y} T_{x^{-1}}\right)=\pi\left(T_{x}\right) \sigma\left(T_{y}\right)
$$

for all $x, y \in W$. Thus

$$
T_{w_{0}}\left[C_{S_{1}}+q^{\frac{1}{2}} c_{\alpha_{1}}(\tau)\right) \cdots\left(C_{S_{r}}+q^{\frac{1}{2}} c_{\alpha_{r}}(\tau)\right]=q^{\frac{r}{2}} \sum_{y \geq w_{0} w} a_{w_{0} w, y}(\tau) T_{y} .
$$

The identity follows.

Finally, the Steinberg representation is a quotient of $I\left(\tau_{0}\right)$ for that $\tau_{0}$ which satisfies $c_{\alpha}\left(\tau_{0}\right)=0$ for all $\alpha \in \Sigma$. 
6. Holomorphicity of the $f_{w}$ 's. We will be interested in the poles of the $a_{w, y}$ 's on $T$. We can get some idea about them from our first formula in (4.2). If we think of $a_{w, y}$ as a polynomial in an indeterminant $q^{-1}$, we see that the degree of $a_{w, y}$ is at most $l(y)-$ $l(w)$. Moreover,

(6.1) Proposition. Let $w_{0}$ be the long word in $W$. For any $w \in$ $W$, the coefficient of $q^{l(w)-l\left(w_{0}\right)}$ in $a_{w, w_{0}}$ is

$$
\prod_{\substack{\alpha>0 \\ w^{-1} \alpha>0}} \frac{e_{\alpha}}{1-e_{\alpha}} \text {. }
$$

Proof. Let $m_{w, y}$ be the coefficient of $q^{l(w)-l(y)}$ in $a_{w, y}$. Then if $\alpha \in \Sigma, s=s_{\alpha}$ and $s w<w, s y<y$, we have

$$
m_{s w, y}=\frac{e_{\alpha}}{1-e_{\alpha}}\left[m_{s w, s y}-\frac{1}{1-e_{\alpha}} s \cdot m_{w, s y}\right] .
$$

On the other hand,

$$
\begin{aligned}
s \cdot m_{w, y} & =s \cdot\left[\frac{e_{\alpha}}{1-e_{\alpha}} m_{w, s y}+s \cdot m_{s w, s y}\right] \\
& =\frac{1}{e_{\alpha}-1} s \cdot m_{w, s y}+m_{s w, s y},
\end{aligned}
$$

so that

$$
m_{s w, u y}=\frac{e_{\alpha}}{1-e_{\alpha}} s \cdot m_{w, y} .
$$

The result now follows by downward induction on $w$.

We are now ready for the main result of this section, which says that (6.1) essentially accounts for all the poles. This determines the domain of holomorphicity of the $f_{w}$ 's.

(6.2) THEOREM. Let $\alpha$ be a positive root and $w \in W$. If $w^{-1} \alpha<0$ then $a_{w, y}$ is holomorphic on $\left\{\tau \in T: e_{\alpha}(\tau)=1\right\}$ for all $y \in W$.

REMARK. The condition means that $w$ is maximal in $W_{\tau} w$ with respect to a certain partial order which is weaker than the Bruhat order. See $\S 8$ and compare with the preliminary result (4.1).

Proof. We can write $a_{w, y}=\frac{P_{y}}{Q_{y}}$ where $P_{y}, Q_{y} \in \mathbb{C}[T]$ are such that $P_{y}$ is not divisible by $e_{\beta}-1$ for any $\beta \in \Delta$, and $Q_{y}$ is a constant multiple of $\prod_{\beta>0}\left(e_{\beta}-1\right)^{m_{\beta}(y)}$ for certain $m_{\beta}(y) \in \mathbb{Z}$. Set 
$m=\max _{y \geq w} m_{\alpha}(y)$. Since $a_{w, w}=1$, we have $m_{\alpha}(w)=0$, and hence $m \geq 0$. The theorem will be proved if we can show that $m=0$. To this end, we suppose $m=m_{\alpha}\left(y_{0}\right)>0$ and we will deduce that $P_{y_{0}}$ vanishes on $V=\left\{\tau \in T: e_{\alpha}(\tau)=1\right\}$. We then argue as follows. Let $\bar{T}$ be a maximal torus in the Langlands dual of the simply connected cover of $G$. We may view $\mathbb{C}[\bar{T}] \subseteq \mathbb{C}[T]$, and from the formulae for $a_{x, y}$, it is clear that $P_{y_{0}} \in \mathbb{C}[\bar{T}]$. Now $\mathbb{C}[\bar{T}]$ is the localization of the polynomial ring $\mathbb{C}\left[e_{\beta}: \beta \in \Sigma\right]$ at powers of $\prod_{\beta \in \Sigma} e_{\beta}$, so the ideal in $\mathbb{C}[\bar{T}]$ generated by $e_{\alpha}-1$ is prime (we may conjugate by $W$ to assume $\alpha$ simple, and then $\left(e_{\alpha}-1\right)$ is clearly prime). It follows that $e_{\alpha}-1$ divides $P_{y_{0}}$ in $\mathbb{C}[\bar{T}]$, hence in $\mathbb{C}[T]$, and this is a contradiction.

Let

$$
U=\left\{\tau \in T: W_{\tau} \subseteq\left\langle s_{\alpha}\right\rangle\right\}
$$

Note that $U \cap V$ is a non-empty open subset of $V$, so it is enough to show that $P_{y_{0}}$ vanishes on $U$.

Let $\tau_{0} \in U$. Then $W_{\tau_{0}} w=\{w\}$ or $\left\{w, s_{\alpha} w\right\}$. In either case, $w$ is maximal in $W_{\tau_{0}} w$. By (4.1), there exists a unique $f_{w}^{0} \in I\left(\tau_{0}\right)_{w} \cap I\left(\tau_{0}\right)^{B}$ transforming under $\Theta$ by $\tau_{0}^{w}$ such that $f_{w}^{0}(w)=1$. On the other hand, for each $g \in G$, the function

$$
\tau \mapsto \sum_{y} a_{w, y}\left(e_{\alpha}-1\right)^{m}(\tau) \varphi_{y}^{\tau}(g)
$$

is holomorphic on $U$ and agrees with $\tau \mapsto\left(e_{\alpha}(\tau)-1\right)^{m} f_{w}^{\tau}(g)$ on $U \cap T^{00}$. Set

$$
F_{w}^{0}(g)=\lim _{\substack{\tau \rightarrow \tau_{0} \\ \tau \in T^{00}}}\left(e_{\alpha}(\tau)-1\right)^{m} f_{w}^{\tau}(g) .
$$

Then $F_{w}^{0} \in I\left(\tau_{0}\right)_{w} \cap I\left(\tau_{0}\right)^{B}$, and transforms under $\Theta$ in the same way as $f_{w}^{0}$. By the uniqueness part of (4.1), we have $F_{w}^{0}=c f_{w}^{0}$ for some constant $c$. Evaluation at $w$ gives

$$
c=\lim _{\tau \rightarrow \tau_{0}}\left(e_{\alpha}-1\right)^{m} a_{w, w}(\tau)=\lim _{\tau \rightarrow \tau_{0}}\left(e_{\alpha}-1\right)^{m}(\tau)=0,
$$

since $m>0$ by assumption. But $F_{w}^{0}$ is a linear combination of $\varphi_{y}^{\tau_{0}}$ 's which are linearly independent. It follows that

$$
\lim _{\substack{\tau \rightarrow \tau_{0} \\ \tau \in T^{00}}}\left(e_{\alpha}-1\right)^{m} a_{w, y}(\tau)=0
$$

for all $y \geq w$. Taking $y=y_{0}$, we find that $P_{y_{0}}\left(\tau_{0}\right)=0$, and this finishes the proof. 
(6.3) Corollary. For $g \in G$, the function $\tau \mapsto f_{w}^{\tau}(g)$ extends to a holomorphic function on $\left\{\tau \in T: w^{-1} \Delta_{\tau}^{+} \subseteq \Delta^{-}\right\}$. For $\tau$ in this domain, we have $f_{w}^{\tau} \in I(\tau)_{w} \cap I(\tau)^{B}$ and $\pi\left(\theta_{a}\right) f_{w}^{\tau}=\tau^{w}(a) f_{w}^{\tau}$ for all $a \in A$.

REMARK. Proposition (6.1) says, at least for generic $q$, that the $f_{w}$ 's do not extend to a larger domain in $T$.

7. The lattice of submodules of $I(\tau), \tau$ regular. Throughout this section, we assume $\tau \in T^{00}$.

Let $S_{\tau}=\left\{\alpha \in \Delta: e_{\alpha}(\tau)=q^{-1}\right\}$. By [R], the irreducible subquotients of $I(\tau)$ are parametrized by subsets of $S_{\tau}$. (Our parametrization is slightly different from that of [R].) If $J \subset S_{\tau}$, the corresponding irreducible subquotient $\pi_{J}$ is characterized by its set of $\Theta$-eigencharacters being $\left\{\tau^{w}: w \in W(J)\right\}$, where

$$
W(J)=\left\{w \in W: S_{\tau} \cap w\left(\Delta^{-}\right)=J\right\} .
$$

Furthermore, $\pi_{J}$ is the unique irreducible submodule of $I\left(\tau^{y}\right)$ if and only if $y \in W(J)$.

Note that $y \in W(J) \Leftrightarrow\left\{\alpha \in S_{\tau}: y^{-1} \alpha<0\right\}=J$.

Let $\tilde{\pi}_{J}$ be the submodule of $I(\tau)$ generated by $\left\{f_{w}^{\tau}: w \in W(J)\right\}$.

(7.1) Proposition. (1) $\pi_{J}$ is the unique irreducible quotient of $\tilde{\pi}_{J}$.

(2) Every G-invariant subspace of $I(\tau)$ is a sum of $\tilde{\pi}_{J}$ 's.

Proof. Let

$$
0 \rightarrow U \rightarrow \tilde{\pi}_{J} \rightarrow V \rightarrow 0
$$

be an exact sequence of $G$ modules, with $V$ irreducible and nonzero. Then $V \simeq \pi_{K}$ for some $K \subseteq S_{t}$. By definition, there is a $w \in W(J)$ such that $f_{w}^{\tau}$ projects nontrivially into $V$. It follows that $\tau^{w}$ appears in $V^{B}$, so $W(J) \cap W(K) \neq \varnothing$, whence $J=K$.

For the second assertion, let $M$ be a $G$-invariant subspace, and suppose $\pi_{J_{1}}, \ldots, \pi_{J_{r}}$ is a complete list of the irreducible quotients of $M$, up to isomorphism. Considering $\Theta$-eigenvectors as above, we see that $\sum_{1 \leq i \leq r} \tilde{\pi}_{J_{i}} \subseteq M$. This containment must be an equality since $I(\tau)$ is multiplicity-free.

Given $J \subseteq S_{\tau}$, we set

$$
R_{J}=\Delta^{+} \cap\left(J \cup-\left(S_{\tau} \backslash J\right)\right) .
$$

We have $y \in W(J)$ if and only if $R_{J}=R\left(y^{-1}\right) \cap\left(S_{\tau} \cup-S_{\tau}\right)$. Next, we define a partial order $\leq$ on the set of subsets of $S_{\tau}$ by

$$
K \leq J \Leftrightarrow R_{K} \subseteq R_{J}
$$


Note that if $S_{t} \subseteq \Delta^{+}$, then $R_{J}=J$ for each $J$ and the partial order $\leq$ is just containment. In general, the minimal and maximal subsets are $S_{\tau} \cap \Delta^{-}$and $S_{\tau} \cap \Delta^{+}$respectively, since

$$
R_{S_{\tau} \cap \Delta^{-}}=\varnothing \quad \text { and } \quad R_{S_{\tau} \cap \Delta^{+}}=\left(S_{\tau} \cap \Delta^{+}\right) \cup-\left(S_{\tau} \cap \Delta^{-}\right) .
$$

A version of the following result, stated in terms of characters in Jacquet modules, appears in [R, Prop. 2].

(7.2) Proposition. Let $J \subset S_{t}$. If $y \in W(J)$, then

$$
\left\{f_{w}^{\tau}: w \in \bigcup_{K \nsupseteq J} W(K)\right\}
$$

is a basis of $\left(\operatorname{ker} \mathscr{A}_{y}\right)^{B}$.

Proof. It is immediate from (4.5) that $\left(\operatorname{ker} \mathscr{A}_{y}\right)^{B}$ is spanned by those $f_{w}^{\tau}$ 's for which there exists a positive root $\alpha \in S_{t} \cup-S_{\tau}$ such that $w^{-1} \alpha>0$ and $y^{-1} \alpha<0$. In other words, $f_{w}^{\tau} \in \operatorname{ker} \mathscr{A}_{y} \Leftrightarrow$ $R\left(y^{-1}\right) \cap\left(S_{\tau} \cup-S_{\tau}\right) \nsubseteq R\left(w^{-1}\right) \cap\left(S_{\tau} \cup-S_{\tau}\right) \Leftrightarrow w \in W(K)$ for some $K \nsupseteq J$.

(7.3) Theorem. Let $J$ and $K$ be two subsets of $S_{\tau}$. Then

$$
\tilde{\pi}_{J} \subseteq \tilde{\pi}_{K} \Leftrightarrow J \leq K .
$$

Proof. Suppose $J \not \leq K$, and take $y \in W(J)$. By (7.2), we know $\tilde{\pi}_{K} \subseteq \operatorname{ker} \mathscr{A}_{y}$. On the other hand, $\mathscr{A}_{y}$ is not identically zero on $\tilde{\pi}_{J}$; hence $\tilde{\pi}_{J} \nsubseteq \tilde{\pi}_{K}$.

Conversely, suppose $J \leq K$, and again choose $y \in W(J)$. Since $\pi_{J}$ is the irreducible quotient of $\tilde{\pi}_{J}$ and is also isomorphic to the irreducible submodule $V$ of $I\left(\tau^{y}\right)$, we must have $\mathscr{A}_{y}\left(\tilde{\pi}_{J}\right)=V$. Now if $w \in W(K)$, we have $\mathscr{A}_{y}\left(f_{w}^{\tau}\right) \neq 0$ so that $\mathscr{A}_{y}\left(\tilde{\pi}_{K}\right)$ is nonzero; hence $V \subseteq \mathscr{A}_{y}\left(\tilde{\pi}_{K}\right)$. This means $\tilde{\pi}_{J} \subseteq \tilde{\pi}_{K}+\operatorname{ker} \mathscr{A}_{y}$. Let $x \in W(J)$. The character $\tau^{x}$ of $\Theta$ does not appear in ker $\mathscr{A}_{y}$, so it must appear in $\tilde{\pi}_{K}$. But then $f_{x}^{\tau} \in \tilde{\pi}_{K}$. Since $\tilde{\pi}_{J}$ is generated by such $f_{x}^{\tau}$ 's, we have $\tilde{\pi}_{J} \subseteq \tilde{\pi}_{K}$.

(7.4) Corollary. (1) The submodule $\tilde{\pi}_{J}^{B}$ has a basis $\left\{f_{w}^{\tau}: w \in\right.$ $W(K)$, where $\left.R_{K} \subseteq R_{J}\right\}$

(2) The G-module $I(\tau)$ has a filtration

$$
0 \subset V_{0} \subset V_{1} \subset \cdots \subset V_{\left|S_{\tau}\right|}=I(\tau)
$$


where

$$
V_{i}=\sum_{\left|R_{J}\right|=i} \tilde{\pi}_{J} \text { and } V_{i} / V_{i-1} \simeq \bigoplus_{\left|R_{J}\right|=i} \pi_{J}
$$

Putting (7.1) and (7.4) together, we have found a basis of every $\widetilde{\mathscr{H}}$ submodule of $I(\tau)^{B}$.

For example, suppose $K \subseteq S_{\tau} \cap \Sigma$. Rogawski has defined a "representation on a parabolic cone"

$$
\mathscr{J}_{K}(\tau)=\bigcap_{\alpha \in K} \operatorname{ker} \mathscr{A}_{S_{\alpha}}
$$

Comparing $\Theta$-characters with the aid of (7.2) and (7.4), we find that

$$
\tilde{\pi}_{S_{\tau}-K}=\mathscr{J}_{K}(\tau)
$$

8. Completeness of our eigenvectors. We return to the setting of an arbitrary $\tau \in T$, but retain the notation $S_{\tau}=\left\{\alpha \in \Delta: e_{\alpha}(\tau)=q^{-1}\right\}$. There is a canonical decomposition

$$
I(\tau)^{B}=\bigoplus_{w \in W_{\tau} \backslash W} I(w, \tau)_{\text {gen }}
$$

where $I(w, \tau)_{\text {gen }}$ is the largest $\Theta$-invariant subspace of $I(\tau)^{B}$ on which the operators $\pi\left(\theta_{a}\right)-\tau^{w}(a), a \in A$, are nilpotent. The dimension of $I(w, \tau)_{\text {gen }}$ is $\left|W_{\tau}\right|$. We also let $I(w, \tau) \subseteq I(w, \tau)_{\text {gen }}$ be the $\Theta$-eigenspace with character $\tau^{w}$.

By (6.3), we have $f_{z}^{\tau} \in I(w, t)$ for all $z \in W_{\tau} w$ with the property that $z^{-1} \Delta_{\tau}^{+} \subseteq \Delta^{-}$. This condition can be reformulated as follows.

First, define a Bruhat-like partial order $\leq^{\tau}$ on $W_{\tau} w$ by declaring that $x w \leq \tau y w$ if and only if there exists a sequence $\alpha_{1}, \ldots, \alpha_{r} \in \Delta_{\tau}^{+}$ such that $s_{\alpha_{r}} \cdots s_{\alpha_{1}} x=y,(x w)^{-1} \alpha_{1}>0$, and $\left(s_{\alpha_{i-1}} \cdots s_{\alpha_{1}} x w\right)^{-1}\left(\alpha_{1}\right)$ $>0$ for all $i=1, \ldots, r$.

The maximal elements in $W_{\tau} w$ with respect to this partial order are precisely those $z$ such that $z^{-1} \Delta_{\tau}^{+} \subseteq \Delta^{-}$. In particular, such elements always exist. Moreover, given one such $z$, we have

$$
\left\{z_{1}: z_{1}^{-1} \Delta_{\tau}^{+} \subseteq \Delta^{-}\right\}=R_{\tau} z,
$$

where $R_{\tau}=\left\{x \in W_{\tau}: x \Delta_{\tau}^{+}=\Delta_{\tau}^{+}\right\}$is the " $R$-group".

Thus $\operatorname{dim} I(w, \tau) \geq\left|R_{\tau}\right|$. We seek conditions for equality. In other words, when does $\left\{f_{z}^{\tau}: z \in W_{\tau} w, z^{-1} \Delta_{\tau}^{+} \subseteq \Delta^{-}\right\} \operatorname{span} I(w, \tau)$ ?

Let

$$
\Delta_{\tau}^{\prime}=\left\{\alpha \in \Delta:\left|e_{\alpha}(\tau)\right|=1\right\}
$$


Note that $\Delta_{\tau}^{\prime}$ is a sub-root system of $\Delta$ containing $\Delta_{\tau}$. We denote the Weyl group of $\Delta_{\tau}^{\prime}$ by $W_{\tau}^{\prime}$.

(8.1) Definition. We say the element $\tau \in T$ is "good" if there exists an intermediate group $W_{\tau} \subseteq W_{0} \subseteq W_{\tau}^{\prime}$ which is generated by $W_{0} \cap\left\{s_{\alpha}: \alpha \in \Sigma\right\}$.

Note that $W_{0}$ is the Weyl group of a root system $\Delta_{0}$ with base $\Delta_{0} \cap \Sigma$. For example, if $W_{\tau}$ is a standard parabolic subgroup of $W$, then $\tau$ is good. Any unitary $\tau$ is also good. However, the advantage of this definition is that for every $\tau \in T$, there exists $w \in W$ such that $\tau^{w}$ is good.

(8.2) LemMA. Let $\tau \in T$.

(1) There exists $w \in W$ such that $\left|e_{\alpha}\left(\tau^{w}\right)\right| \leq 1$ for all $\alpha>0$. For such a $w, \tau^{w}$ is good.

(2) If $\tau$ is good, then $\tau^{w}$ is also good for all $w \in W_{0}$.

Proof. The assertion (2) is clear. For (1), we observe that there exists $w \in W$ such that

$$
w \Delta^{+}=\left\{\alpha \in \Delta:\left|e_{\alpha}(\tau)\right|<1\right\} \cup\left(\Delta_{\tau}^{\prime} \cap \Delta^{+}\right),
$$

because the right side is a positive system for $\Delta$, whence the first part of (1). For the second part, suppose $\alpha \in \Delta_{\tau}^{\prime} \cap \Delta^{+}$and $\alpha=\alpha_{1}+\cdots+\alpha_{n}$ where each $\alpha_{i}$ belongs to $w \Sigma$. Then $1=\left|e_{\alpha_{1}+\cdots+\alpha_{n}}(\tau)\right|=\prod\left|e_{\alpha_{i}}(\tau)\right|$. But the choice of $w$ insures that $\left|e_{\alpha_{i}}(\tau)\right| \leq 1$ for each $i$. Hence for all $i,\left|e_{\alpha_{i}}(\tau)\right|=1$ so $\alpha_{i} \in \Delta_{\tau}^{\prime}$. It follows that $W_{\tau}^{\prime}$ is generated by the simple reflections it contains, so $\tau^{w}$ is good.

We are now ready to state the main result of this section.

(8.3) Theorem. (1) If $\tau$ is good, then $\operatorname{dim} I(e, \tau)=\left|R_{\tau}\right|$.

(2) If $R_{\tau}$ is trivial and $\tau^{w}$ is $g o o d$, then $\operatorname{dim} I(w, \tau)=\left|R_{\tau}\right|=1$.

(3) Suppose $y \in W$ and $R\left(y^{-1}\right) \cap \pm S_{\tau}=\varnothing$. Then for all $w \in W$, $I\left(w^{-1}, \tau^{w}\right) \simeq I\left(y w^{-1}, \tau^{w}\right)$ and $I\left(w, \tau^{y}\right) \simeq I(y w, \tau)$.

In particular, if $S_{\tau}=\varnothing$ then $\operatorname{dim} I\left(y, \tau^{w}\right)=\left|R_{\tau}\right|$ for all $y, w \in W$.

Proof. The dimension of the space of $\Theta$-eigenvectors in $I(w, \tau)$ equals the number of $\Theta$-indecomposable summands in $I(w, \tau)$, which in turn is the dimension of $\operatorname{Hom}_{\Theta}\left(I(\tau), \tau^{w}\right) \simeq \operatorname{Hom}_{G}\left(I(\tau), I\left(\tau^{w}\right)\right)$. If $\tau$ is unitary, it follows from a well-known theorem of Harish-Chandra (see [S1]) that the dimension of this last space is at most $\left|R_{\tau}\right|$. This finishes the proof in this case. 
We now let $\tau \in T, w \in W$ be such that $\tau^{w}$ is good. Let $W_{0}, \Delta_{0}$ be as in (8.1), applied to $\tau^{w}$. Consider the subgroup $G_{0}$ of $G$ generated by the roots from $\Delta_{0}$. Since $G_{0}$ is again a Chevalley group (with Weyl group $W_{0}$ ), we have an Iwahori-Hecke algebra $\mathscr{C}_{0}$ for $G_{0}$. We may identify $\mathscr{H}_{0}$ with the subalgebra of $\mathscr{H}$ generated by the elements $T_{s_{\alpha}}$ and $\Theta_{0}$, where $\Theta_{0}$ is the linear span of $\left\{\theta_{a}: a \in A \cap G_{0}\right\}$. Let $\tau_{0}$ be the restriction of $\tau$ to $A \cap G_{0}$. Note that $\tau_{0}$ is unitary.

(8.4) LEMMA. Under either of the hypotheses in (8.3)(1) or (2), there exists $z \in W_{\tau^{w}}$ with $z^{-1} \Delta_{\tau}^{+} \subseteq \Delta^{-}$such that the $\mathscr{H}_{0}$-module $\mathscr{H}_{0} f_{z}^{\tau}$ is isomorphic to the induced (principal series) representation $\mathscr{H}_{0} \otimes_{\Theta_{0}} \tau_{0}$.

Proof. We have a surjective $\mathscr{H}_{0}$-homomorphism

$$
\mathscr{H}_{0} \otimes_{\boldsymbol{\Theta}_{0}} \tau_{0} \rightarrow \mathscr{H}_{0} f_{z}^{\tau}
$$

such that $T_{y} \otimes 1 \mapsto \pi\left(T_{y}\right) f_{z}^{\tau}$. If $R_{\tau}$ is trivial, this map is injective because the induced module is irreducible (cf. [K]).

Suppose $\tau$ is good and $w=e$. Let $z$ be the long word of $W_{0}$. By (4.9), we have

$$
\pi\left(T_{y^{-1}}\right) f_{z}^{\tau}=q^{l(y)}\left(\prod_{\alpha \in R(y)} J_{\alpha, z}\right) f_{z y}^{\tau}+\sum_{x<y} c_{x} f_{z x}^{\tau},
$$

for some complex numbers $c_{x}$. Since $W_{0}$ is generated by simple reflections, $x<y$ is equivalent to $z y<z x$. Thus

$$
\pi\left(T_{y^{-1}}\right) f_{z}^{\tau}=q^{l(y)} \varphi_{z y}+\sum_{x<y} b_{x} \varphi_{z x}
$$

implying that $\left\{\pi\left(T_{y}\right) f_{z}^{\tau}: y \in W_{0}\right\}$ is linearly independent.

We can now finish the proofs of $(8.3)(1)$ and (2). Since $\mathscr{H}_{0} f_{z}^{\tau}$ is a unitary principal series representation of $\mathscr{H}_{0}$, it follows that $\mathscr{H}_{0} f_{z}^{\tau}$ has $\left[W_{0}: W_{\tau^{w}}\right]$ generalized eigenspaces for $\Theta_{0}$, each of dimension $\left|W_{\tau}\right|$ and there are exactly $\left|R_{\tau}\right| \Theta_{0}$-indecomposable summands in each generalized eigenspace. On the other hand, $I(w, \tau)_{\text {gen }}$ also has dimension $\left|W_{\tau}\right|$. It follows that $I(w, \tau)_{\text {gen }}$ is a generalized eigenspace for $\Theta_{0}$ in $\mathscr{L}_{0} f_{z}^{\tau}$ and hence there are at most $\left|R_{\tau}\right|$ indecomposable summands for $\Theta$ in $I(w, \tau)$.

For (3), we may assume $y=s_{\alpha}$ is a simple reflection and $e_{\alpha}(\tau) \neq 1$. By (4.8) the operator

$$
\pi\left(T_{s}\right)-q+q c_{\alpha}(\tau)
$$


maps the eigenspaces in $I\left(\tau^{w}\right)$ for characters $\tau$ and $\tau^{s}$ into each other. The possible eigenvalues of $T_{s}$ are -1 and $q$, so this operator is injective if $\alpha \notin \pm S_{\tau}$.

The second isomorphism results from the fact that the normalized intertwining operator $\widetilde{\mathscr{A}_{s}} \in \operatorname{Hom}_{G}\left(I(\tau), I\left(\tau^{s_{\alpha}}\right)\right)$ is an isomorphism if $\alpha \notin \pm S_{\tau}$. (Though well known, this also follows from (4.10).)

REMARK. One can show, in the notation of the above proof, that

$$
\mathscr{H}_{0} f_{z}^{\tau}=\bigoplus_{x \in W_{\tau} \backslash W_{0}} I(x, \tau)
$$

(8.6) CoRollary. Suppose $\tau$ is good and $R_{\tau}$ is trivial. Then $I(\tau)$ has a unique irreducible quotient and submodule, and these occur with multiplicity one.

Proof. Note that $\tau$ is good if and only if $\tau^{w_{0}}$ is good. Since $R_{\tau}=1$, $(8.3)(2)$ implies that $I(w, \tau)$ is spanned by $f_{w_{0}}^{\tau}$, which we know to be a generator of $I(\tau)^{B}$. It follows that the sum of all submodules not containing $f_{w_{0}}^{\tau}$ is the unique maximal proper submodule of $I(\tau)$, and the isomorphism class of the quotient has multiplicity one. The hypotheses are the same for $\tau^{-1}$, and $I\left(\tau^{-1}\right)$ is the contragredient representation of $I(\tau)$, so $I(\tau)$ also has a unique irreducible submodule with multiplicity one.

The examples in $\S 9$ show that even for $S L_{3}$, neither hypothesis can be omitted.

(8.7) Corollary (Müller, Kato). I $I$ ) is irreducible if and only if $S_{t}=\varnothing$ and $R_{\tau}=1$.

Proof. We know the dimension of $\operatorname{End}_{\mathscr{H}}\left(I(\tau)^{B}\right)$ is at least $\left|R_{\tau}\right|$, from our construction of $\Theta$-eigenvectors, so irreducibility implies $R_{\tau}$ $=1$. Suppose $S_{\tau}$ is non-empty. Conjugating $\tau$ if necessary, we may assume $S_{\tau}$ contains a simple root $\alpha$. As in $\S 4$, let $\bar{\alpha}=-w_{0} \alpha \in \Sigma$. Since $w_{0} s_{\alpha} \Delta_{\tau}^{+} \subseteq \Delta^{-}$, and $J_{\bar{\alpha}, w_{0} s_{\alpha}}=c_{\alpha} c_{-\alpha}(\tau)=0$, (4.8) says that $f_{s_{\alpha} w_{0}}^{\tau}$ is an eigenvector for $T_{S_{\bar{\alpha}}}$. It follows that $f_{s_{\alpha} w_{0}}^{\tau}$ is contained in a submodule induced from a character on nonminimal parabolic subalgebra of $\mathscr{H}$. This submodule has dimension equal to the index of the corresponding parabolic subgroup of $W$, hence is proper.

Suppose $M$ is a nonzero submodule of $I(\tau)^{B}$. Since $S_{t}=\varnothing,(4.8)$ implies that $M$ contains $\Theta$-eigenvectors for every character in the $W$-orbit of $\tau$, and the dimensions of all $\Theta$-eigenspaces are equal. 
Since there exists $w \in W$ such that $\tau^{w}$ is good, (8.3) implies that $\operatorname{dim} I(w, \tau)=\left|R_{\tau}\right|=1$ for all $w \in W$. It follows that $M$ contains all $\Theta$-eigenvectors. In particular, $f_{w_{0}}^{\tau} \in M$, so $M=I(\tau)^{B}$.

9. Examples. First, suppose $\tau$ is unitary. Then $I(\tau)$ is completely reducible. Recall that we have a homomorphism $r \mapsto \widetilde{\mathscr{A}}_{r}$ of the group $R_{\tau}$ into the invertible elements in $\operatorname{End}_{G}(I(\tau))=\operatorname{End}_{\mathscr{H}}\left(I\left(\tau^{B}\right)\right)$, where $\widetilde{\mathscr{A}_{r}}$ is a normalized intertwining operator. This extends to a ring isomorphism $\mathbb{C} R_{\tau} \simeq \operatorname{End}_{\mathscr{H}}\left(I(\tau)^{B}\right)$. Thus we have a decomposition $I(\tau)^{B}=\bigoplus_{\eta \in \widehat{R}_{\tau}} V_{\eta}$ into isotypic components. Each $V_{\eta}$ is a direct sum of $\operatorname{dim} \eta$ pairwise isomorphic $\mathscr{H}$-modules. We will describe the space of $\Theta$-eigenvectors in each $V_{\eta}$.

We identify $\eta$ with its character and let

$$
\Pi_{\eta}=\sum_{r \in R_{\tau}} \bar{\eta}(r) \widetilde{\mathscr{A}_{r}} \in \operatorname{End}\left(I(\tau)^{B}\right)
$$

be the projection onto $V_{\eta}$. Let $w \in W$, and choose $w_{1} \in W_{\tau^{w}}$ such that $w_{1}^{-1} \Delta_{\tau} \subseteq \Delta^{-}$. It follows from (4.2) that the linear span of $\left\{f_{r w_{1}}^{\tau}: r \in R_{\tau}\right\}$ is invariant under $R_{\tau}$, and in fact affords the regular representation of $R_{\tau}$. This implies that

$$
\Pi_{\eta}\left\langle f_{r w_{1}}^{\tau}: r \in R_{\tau}\right\rangle
$$

has dimension $(\operatorname{dim} \eta)^{2}$ and is the linear span of the $\Theta$-eigenvectors in $V_{\eta}$ which transform by $\tau^{w}$.

Here is a pleasing example of this. Let

$$
G=S L_{n} \quad \text { and } \quad \tau=\operatorname{diag}\left(1, \omega, \omega^{2}, \ldots, \omega^{n-1}\right) \in P G L_{n}(\mathbb{C}),
$$

where $\omega$ is a primitive $n$th root of unity. Then $R_{\tau}=W_{\tau}=\langle\sigma\rangle$ is cyclic of order $n$. All $f_{w}^{\tau}$ are holomorphic, $\Theta$ is diagonalizable on $I(\tau)^{B}$ and each $V_{\eta}$ is irreducible for $\mathscr{H}$. Choose a set of coset representatives $\Omega$ for $R_{\tau} \backslash W$. Then $\left\{\Pi_{\eta} f_{w}^{\tau}: w \in \Omega\right\}$ is a basis of $V_{\eta}$ consisting of $\Theta$-eigenvectors. In particular, $\operatorname{dim} V_{\eta}=(n-1)$ !. Moreover, using (4.2) we see that

$$
\Pi_{\eta} f_{w}^{\tau}=\sum_{i=0}^{n-1} \frac{1}{c_{\sigma}^{i}} \prod_{\alpha \in R\left(\sigma^{l}\right)} K_{\alpha, w} f_{\sigma^{-l} w}^{\tau}
$$

We should also discuss at least one group thoroughly. Take $G=$ $S L_{3}(F)$, with $\Sigma=\{\alpha, \beta\}$. Set $s=s_{\alpha}, r=s_{\beta}$. The torus $T$ may be viewed as $\left(\mathbb{C}^{\times}\right)^{3}$ modulo the diagonal action of $\mathbb{C}^{\times}$, such that if $\tau \in T$ is represented by $\left(x_{1}, x_{2}, x_{3}\right)$, then $e_{\alpha}(\tau)=x_{1} x_{2}^{-1}, e_{\beta}(\tau)=x_{2} x_{3}^{-1}$. 
Then in $\widetilde{\mathscr{H}}$ we have

$$
\begin{aligned}
f_{s r s}= & T_{s r s}, \\
f_{r s}= & T_{r s}+\left(1-c_{\alpha}\right) T_{s r s}, \quad f_{s r}=T_{s r}+\left(1-c_{\beta}\right) T_{s r s}, \\
f_{r}= & T_{r}+\left(1-c_{\alpha}\right) T_{s r}+\left(1-c_{\alpha+\beta}\right) T_{r s}+\left(1-c_{\alpha}\right)\left(1-c_{\alpha+\beta}\right) T_{s r s}, \\
f_{s}= & T_{s}+\left(1-c_{\beta}\right) T_{r s}+\left(1-c_{\alpha+\beta}\right) T_{s r}+\left(1-c_{\beta}\right)\left(1-c_{\alpha+\beta}\right) T_{s r s}, \\
f_{e}= & T_{e}+\left(1-c_{\alpha}\right) T_{s}+\left(1-c_{\beta}\right) T_{r}+\left(1-c_{\alpha}\right)(1-c \beta)\left(T_{r s}+T_{s r}\right) \\
& +\left[\left(1-c_{\alpha}\right)\left(1-c_{\beta}\right)\left(1-c_{\alpha+\beta}\right)+q^{-1}\left(1-c_{\alpha+\beta}\right)\right] T_{s r s} .
\end{aligned}
$$

It is useful to know that

$$
1-c_{\alpha+\beta}=\frac{\left(1-c_{\alpha}\right)\left(1-c_{\beta}\right)}{\left(1-c_{\alpha}\right)+\left(q^{-1}-c_{\beta}\right)} .
$$

We will find bases of $I(w, \tau)_{\text {gen }}$ for each $\tau \in T, w \in W$, and relate them to the $\mathscr{H}$ module structure on $I(\tau)^{B}$.

First, if $\Delta_{\tau}=\varnothing$, then either $W_{\tau}=1$ or $\tau$ is $W$-conjugate to $\left(1, \omega, \omega^{2}\right)$, and these cases have been covered in (7.3) and above. If $\Delta_{\tau}=\Delta$ then $\tau=1, \Theta$ acts indecomposably, $f_{\text {srs }}^{1}$ is the only $\Theta$ eigenvector and $I(1)^{B}$ is irreducible.

Thus we arrive at the cases where $\Delta_{\tau}^{+}$consists of a single root. Here $R_{\tau}$ is trivial.

For each positive root $\gamma$, set $U_{\gamma}=\left\{\tau \in T: \Delta_{\tau}^{+} \subseteq\{\gamma\}\right\}$. The $U_{\gamma}$ 's form an open cover of $T \backslash\{1\}$. One can verify directly that if $\tau \in U_{\gamma}$, and $w^{-1} \gamma<0$, then $f_{w}$ and $f_{s_{y} w}-a_{s_{\gamma}} w, w f_{w}$ belong to $\mathbb{C}[T]_{\tau} \otimes \mathscr{H}_{W}$. Set $h_{w}^{\tau}=\mathscr{P}_{\tau} \mu_{\tau}\left(f_{s_{y} w}-a_{s_{\gamma} w, w} f_{w}\right)$. Then

$$
I(w, \tau)_{\mathrm{gen}}=\left\langle f_{w}^{\tau}, h_{w}^{\tau}\right\rangle
$$

We have, for $\tau \in U_{\gamma} \cap T^{0}$,

$$
\begin{aligned}
& \pi\left(\theta_{a}\right) f_{w}^{\tau}=\tau^{w}(a) f_{w}^{\tau}, \quad \text { and } \\
& \pi\left(\theta_{a}\right) h_{w}^{\tau}=\tau^{s_{\gamma} w}(a) h_{w}^{\tau}+a_{s_{\gamma} w, w}(\tau)\left(\tau^{s_{\gamma}} w(a)-\tau^{w}(a)\right) f_{w}^{\tau} .
\end{aligned}
$$

In fact, this last matrix coefficient is holomorphic on all of $U_{\gamma}$. To see this, we take $a=h_{\delta}(\varpi)$ for some $\delta \in \Delta$. Let $($,$) denote a$ $W$-invariant pairing on $\Delta$, and set $\check{\nu}=(\nu, \nu)^{-1} \nu$, for $\nu \in \Delta$. We then have

$$
\begin{array}{r}
a_{s_{\gamma} w, w}(\tau)\left(\tau^{s_{\gamma} w}\left(h_{\delta}(\varpi)\right)-\tau^{w}\left(h_{\delta}(\varpi)\right)\right) \\
=a_{s_{\gamma} w, w}(\tau) e_{w \delta}(\tau)\left(e_{\gamma}^{-(w \delta, \check{\gamma})}-1\right) .
\end{array}
$$

In all cases except $\gamma=\alpha+\beta, w=s r s$, this last is

$$
\left(1-q^{-1}\right) e_{\gamma} e_{w \delta}\left(\frac{1-e_{\gamma}^{-(w \delta, \check{\gamma})}}{1-e_{\gamma}}\right)(\tau),
$$


which becomes

$$
\left(q^{-1}-1\right)(w \delta, \check{\gamma}) e_{w \delta}(\tau)
$$

on $e_{\gamma}(\tau)=1$. Note that this is never zero, so each $I(w, \tau), w^{-1} \gamma<$ 0 , is spanned by $f_{w}^{\tau}$.

If $\gamma=\alpha+\beta$ and $w=s r s$, the last matrix coefficient is instead

$$
\left[\left(1-c_{\alpha}\right)\left(1-c_{\beta}\right)+q^{-1}\right]\left(1-q^{-1}\right) e_{\alpha+\beta} e_{w \delta}\left(\frac{1-e^{-\left(w \delta,(\alpha+\beta)^{\vee}\right)}}{1-e_{\alpha+\beta}}\right)
$$

which tends to

$$
c_{\alpha} c_{-\alpha}(\tau)\left(q^{-1}-1\right)\left(w \delta,(\alpha+\beta)^{\vee}\right) e_{w \delta}(\tau)
$$

on $e_{\alpha+\beta}=1$. This is nonzero unless $\tau=\left(1, q^{ \pm 1}, 1\right)$, in which case $h_{s r s}^{\tau}=f_{e}^{\tau}-a_{e, s r s} f_{s r s}^{\tau}$ is a fourth $\Theta$-eigenvector, along with $f_{s r s}^{\tau}, f_{s r}^{\tau}$, $f_{r s}^{\tau}$.

To find the $\mathscr{H}$-invariant submodules, we use the relation from (4.9):

$$
\pi\left(T_{s}\right) f_{w}^{\tau}=\left(1-c_{w \alpha}(\tau)\right) f_{w}^{\tau}+J_{\alpha, w} f_{w s}^{\tau}
$$

(and similarly for $r=s_{\beta}$ ), as well as the fact that $f_{w_{0}}^{\tau}$ always generates $I(\tau)^{B}$.

We have six remaining possibilities for reducible $I(\tau)^{B}$.

If $\tau=\left(1,1, q^{ \pm 1}\right) \in U_{\beta}$, there is one $\mathscr{H}$-invariant subspace:

$$
\left\langle f_{s r}^{\tau}, f_{s}^{\tau}, h_{s}^{\tau}\right\rangle
$$

Since $f_{s r s}^{\tau}$ is the only $\Theta$-eigenvector outside this subspace, we see that $I(\tau)^{B}$ has length two, and is indecomposable by (8.6).

If $\tau=\left(q^{ \pm 1}, 1,1\right)$, the picture is the same with $r$ and $s$ interchanged.

If $\tau=\left(1, q^{ \pm 1}, 1\right)$, then $I(\tau)^{B}$ splits into two irreducibles of dimension three:

$$
I(\tau)^{B}=\left\langle f_{s r}^{\tau}, h_{s r}^{\tau}, q h_{s r s}^{\tau}-f_{s r s}^{\tau}\right\rangle \oplus\left\langle f_{r s}^{\tau}, h_{r s}^{\tau}, q h_{s r s}^{\tau}+q^{-1} f_{s r s}^{\tau}\right\rangle .
$$

(These facts are easiest to check by writing each vector in terms of $\varphi_{y}$ 's and checking the action of $T_{r}$ and $T_{s}$. )

Note that the indecomposability of $I(\tau)$ may change throughout the $W$ orbit of $\tau$.

For a general group, it is reasonable to make the following

(9.1) Conjecture. For $\tau \in T$ and $w \in W$, let

$$
\widetilde{\mathscr{H}}_{(w, \tau)}=\mathbb{C}(T)\left\langle f_{x w}: x \in W_{\tau}\right\rangle \cap \mathbb{C}[T]_{\tau} \mathscr{H}_{W},
$$


and recall the map

$$
\mathscr{P}_{\tau} \mu_{\tau}: \mathbb{C}[T]_{\tau} \mathscr{H}_{W} \rightarrow I(\tau)^{B}
$$

which sends $a T_{y}$ to $a(\tau) \varphi_{y}$. Then

$$
I(w, \tau)_{\text {gen }}=\mathscr{P}_{\tau} \mu_{\tau}\left(\widetilde{\mathscr{H}}_{(w, \tau)}\right) .
$$

\section{REFERENCES}

[B] A. Borel, Admissible representations of a semisimple p-adic group over a local field with vectors fixed under an Iwahori subgroup, Inv. Math., 35 (1976), 233-259.

[C] W. Casselman, The unramified principal series of p-adic groups I, Comp. Math., 40 (1980), 387-406.

[G] V. Ginsburg, Deligne-Langlands conjecture and representations of affine Hecke algebras, preprint.

[Ka] S. Kato, Irreducibility of principal series representations for Hecke algebras of affine type, J. Fac. Sci., U. of Tokyo, Sec. 1A, 28 (1982), 929-943.

[K-L] D. Kazhdan and G. Lusztig, Proof of the Deligne-Langlands conjecture for Hecke algebras, Invent. Math., 87 (1987), 153-215.

[K-L1] _ Representations of Coxeter groups and Hecke algebras, Invent. Math., 53 (1979), 165-184.

[K] C. D. Keys, On the decomposition of reducible principal series representations of p-adic Chevalley groups, Pacific J. Math., 101 (1982), 351-388.

[L] G. Lusztig, Affine Hecke algebras and their graded version, J. Amer. Math. Soc., 2 (1989), 599-635.

[M] I. Müller, Integrales d'entrelacement pour un groupe de Chevalley sur un corps p-adique, in "Non-commutative harmonic analysis, Marseille-Luminy," Springer Verlag, Lecture Notes No. 739, Berlin, 1981.

[M2] __ Integrales d'entrelacement pour $G L_{n}(k), k$ p-adique, in Analyse Harmonique sur les groups de Lie, Springer Verlag, Lecture Notes No. 497, Berlin, (1975), 277-347.

[R] F. Rodier, in Non-commutative harmonic analysis, Marseille-Luminy, Springer Verlag, Lecture Notes No. 880, Berlin, 1980.

[Ro] J. Rogawski, On modules over the Hecke algebra of a p-adic group, Invent. Math., 79 (1985), 443-465.

[S] A. Silberger, The Knapp-Stein dimension theorem for p-adic groups, Proc. Amer. Math. Soc., 68 (1978), 243-246.

[S1] _ Introduction to Harmonic Analysis on Reductive p-adic Groups, Princeton Univ. Press, Princeton, N.J., 1979.

Received September 14, 1990.

UNIVERSITY OF OKLAHOMA

NORMAN, OK 73019 


\section{PACIFIC JOURNAL OF MATHEMATICS EDITORS}

\author{
V. S. VARADARAJAN \\ (Managing Editor) \\ University of California \\ Los Angeles, CA 90024-1555-05 \\ Herbert Clemens \\ University of Utah \\ Salt Lake City, UT 84112 \\ F. Michael Christ \\ University of California \\ Los Angeles, CA 90024-1555 \\ THOMAS ENRIGHT \\ University of California, San Diego \\ La Jolla, CA 92093
}

Nicholas ERCOLANI

University of Arizona

Tucson, AZ 85721

R. FINN

Stanford University

Stanford, CA 94305

VAUGHAN F. R. JONES

University of California

Berkeley, CA 94720

Steven Kerckhoff

Stanford University

Stanford, CA 94305

\section{C. MOORE}

University of California

Berkeley, CA 94720

Martin ScharlemanN

University of California

Santa Barbara, CA 93106

HAROLD STARK

University of California, San Diego

La Jolla, CA 92093

\section{R. ARENS \\ ASSOCIATE EDITORS}

\begin{tabular}{|c|c|c|c|c|}
\hline R. Arens & $\begin{array}{l}\text { E. F. BECKENBACH } \\
(1906-1982)\end{array}$ & B. H. NeumanN & $\begin{array}{c}\text { F. WoLF } \\
(1904-1989)\end{array}$ & K. YoshidA \\
\hline \multicolumn{5}{|c|}{ SUPPORTING INSTITUTIONS } \\
\hline \multicolumn{2}{|c|}{ UNIVERSITY OF ARIZONA } & UNIVERSI & OF OREGON & \\
\hline \multicolumn{2}{|c|}{ UNIVERSITY OF BRITISH COLUMB } & & & \\
\hline \multicolumn{2}{|c|}{ CALIFORNIA INSTITUTE OF TECHNOLOGY } & STANF & JNIVI & \\
\hline \multicolumn{2}{|c|}{ UNIVERSITY OF CALIFORNIA } & UNIV & & \\
\hline \multicolumn{2}{|c|}{ MONTANA STATE UNIVERSITY } & UNIVE & OF T & \\
\hline \multirow{2}{*}{\multicolumn{2}{|c|}{ UNIVERSITY OF NEVADA, RENO }} & UNIVE & OF U & \\
\hline & & WASHII & N STATE UN & ERSIT \\
\hline \multicolumn{2}{|c|}{$\begin{array}{l}\text { NEW MEXICO STATE UNIVERSITY } \\
\text { OREGON STATE UNIVERSITY }\end{array}$} & UNIVERS & OF WASHIN & \\
\hline
\end{tabular}

The Supporting Institutions listed above contribute to the cost of publication of this Journal, but they are not owners or publishers and have no responsibility for its content or policies.

Mathematical papers intended for publication in the Pacific Journal of Mathematics should be in typed form or offset-reproduced (not dittoed), double spaced with large margins. Please do not use built up fractions in the text of the manuscript. However, you may use them in the displayed equations. Underline Greek letters in red, German in green, and script in blue. The first paragraph must be capable of being used separately as a synopsis of the entire paper. In particular it should contain no bibliographic references. Please propose a heading for the odd numbered pages of less than 35 characters. Manuscripts, in triplicate, may be sent to any one of the editors. Please classify according to the 1991 Mathematics Subject Classification scheme which can be found in the December index volumes of Mathematical Reviews. Supply name and address of author to whom proofs should be sent. All other communications should be addressed to the managing editor, or Elaine Barth, University of California, Los Angeles, California 90024-1555-05.

There are page-charges associated with articles appearing in the Pacific Journal of Mathematics. These charges are expected to be paid by the author's University, Government Agency or Company. If the author or authors do not have access to such Institutional support these charges are waived. Single authors will receive 50 free reprints; joint authors will receive a total of 100 free reprints. Additional copies may be obtained at cost in multiples of 50 .

The Pacific Journal of Mathematics (ISSN 0030-8730) is published monthly except for July and August. Regular subscription rate: $\$ 190.00$ a year (10 issues). Special rate: $\$ 95.00$ a year to individual members of supporting institutions.

Subscriptions, orders for numbers issued in the last three calendar years, and changes of address should be sent to Pacific Journal of Mathematics, P.O. Box 969, Carmel Valley, CA 93924, U.S.A. Old back numbers obtainable from Kraus Periodicals Co., Route 100, Millwood, NY 10546.

The Pacific Journal of Mathematics at P.O. Box 969, Carmel Valley, CA 93924 (ISSN 0030-8730) is published monthly except for July and August. Second-class postage paid at Carmel Valley, California 93924, and additional mailing offices. Postmaster: send address changes to Pacific Journal of Mathematics, P.O. Box 969, Carmel Valley, CA 93924.

PUBLISHED BY PACIFIC JOURNAL OF MATHEMATICS, A NON-PROFIT CORPORATION Copyright (C) 1992 by Pacific Journal of Mathematics 


\section{Pacific Journal of Mathematics}

Vol. 153, No. $2 \quad$ April, 1992

R. Ayala, Eladio Domínguez Murillo, Alberto Márquez Pérez and A.

Quintero, Lusternik-Schnirelmann invariants in proper homotopy

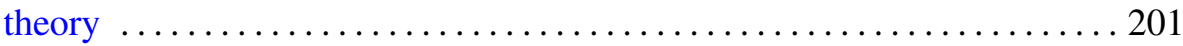

Hari Bercovici and Dan-Virgil Voiculescu, Lévy-Hinčin type theorems for multiplicative and additive free convolution $\ldots \ldots \ldots \ldots \ldots \ldots \ldots 217$

L. J. Bunce and Cho-Ho Chu, Compact operations, multipliers and Radon-Nikodým property in $J B^{*}$-triples $\ldots \ldots \ldots \ldots \ldots \ldots \ldots \ldots \ldots \ldots \ldots \ldots$

Marius Dadarlat, Gabriel Nagy, András Némethi and Cornel Pasnicu, Reduction of topological stable rank in inductive limits of $C^{*}$-algebras

François Dumas and Robert Vidal, Dérivations, et hautes dérivations, dans certains corps gauches de series de Laurent .................... 277

Mourad Ismail and Xin Li, On sieved orthogonal polynomials. IX:

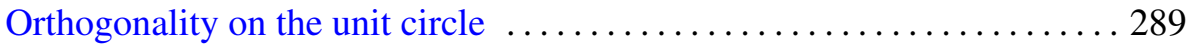

X. T. Liang and Y. W. Lu, A Phragmén-Lindelöf theorem ...............299

Mark Stephen Reeder, On certain Iwahori invariants in the unramified principal series

Shohei Tanaka, On the representation of the determinant of Harish-Chandra's $C$-function of $\operatorname{SL}(n, \mathbb{R})$

Fritz von Haeseler and Guentcho Svetoslavov Skordev, Borsuk-Ulam theorem, fixed point index and chain approximations for maps with multiplicity 